
\title{
Research Suatere \\ Single Base Station Positioning based on Multipath Parameter Clustering in NLOS Environment
}

\section{Yong Wang}

Chongqing University of Posts and Telecommunications

Qihong Wu ( $\square$ wu.qihong@foxmail.com )

Chongqing University of Posts and Telecommunications

Mu Zhou

Chongqing University of Posts and Telecommunications

\section{Xiaolong Yang}

Chongqing University of Posts and Telecommunications

\section{Wei Nie}

Chongqing University of Posts and Telecommunications

\section{Liangbo Xie}

Chongqing University of Posts and Telecommunications

\section{Research Article}

Keywords: Single base station positioning, NLOS, Multipath signal, Parameter clustering, LevenbergMarquardt algorithm

Posted Date: February 16th, 2021

DOl: https://doi.org/10.21203/rs.3.rs-189732/v1

License: (c) (1) This work is licensed under a Creative Commons Attribution 4.0 International License.

Read Full License 


\title{
RESEARCH
}

\section{Single base station positioning based on multipath parameter clustering in NLOS environment}

\author{
Yong Wang, Qihong Wu, Mu Zhou, Xiaolong Yang ${ }^{*}$, Wei Nie and Liangbo Xie
}

\author{
${ }^{*}$ Correspondence: \\ yangxiaolong@cqupt.edu.cn \\ School of Communication and \\ Information Engineering, \\ Chongqing University of Posts and \\ Telecommunications, Chongqing, \\ China \\ Full list of author information is \\ available at the end of the article
}

\begin{abstract}
This paper proposes a scattering area model based on the spatial layout and a joint clustering algorithm for processing multipath parameters extracted from the model. First of all, we construct a scatter area model based on the spatial layout of obstacles near the base station and then collect the multipath signals needed for positioning and extract parameters. Second, we use the joint clustering algorithm improved by k-means clustering and mean shift clustering algorithm to process the parameters and extract useful information. Third, the processed information is combined with the spatial layout information of the scattering area model to construct equations, and then the solving problem of equations is converted into a least-squares optimization problem. Finally, the Levenberg-Marquardt (LM) algorithm is used to solve the optimal solution and estimate the mobile target position. The simulation results show that the positioning algorithm in this paper can use a single base station to locate the target in an outdoor Non-line-of-sight (NLOS) environment, and the accuracy is improved compared with the traditional positioning algorithm.
\end{abstract}

Keywords: Single base station positioning; NLOS; Multipath signal; Parameter clustering; Levenberg-Marquardt algorithm

\section{Introduction}

Outdoor wireless positioning is a practical function in people's daily life, which has been involved in many aspects of life, and it is also one of the basic service and application modules of government and commercial units, even in the military field. Many companies and scientific research teams are engaged in related research, which is one of the current research hotspots[1, 2]. At present, the mature outdoor wireless positioning technology is based on satellites, such as the Global Positioning System (GPS) and the BeiDou Navigation Satellite System (BDS). However, some shortcomings exist in the satellite-based positioning system. Firstly, the cost of using the satellite is very expensive. Secondly, at least four satellites are required to get target positions accurately. Thirdly, environmental factors have a great impact on localization accuracy[3], such as the building or terrain-blocking signal, the extreme weather environment and geomagnetic storms, all of which may could lead to large positioning errors.

The outdoor positioning technology based on a single communication base station is cost-efficient and convenient for users[4]. It only needs to extract the parameter information from the electromagnetic wave signal of the mobile terminal 
in the communication process to operate. In 1996, the Federal Communications Commission (FCC) issued the Enhanced 911 (E911)[5], which claimed that wireless cellular networks must be able to provide the Location Based Service (LBS) to mobile terminals, so the loclization technology based on cellular base stations has been the focus of research. In the cellular base station based positioning system, the signal parameters from the mobile terminal are extracted for positioning. The commonly-used measurement parameters include the Time of Arrival (TOA), Time of Flight (TOF), Angle of Arrival (AOA), Time Difference of Arrival (TDOA), and Received Signal Strength (RSS) $[6,7]$. By considering the accuracy of signal measurement and parameter extraction, the research early is often based on single parameters. Generally, positioning systems based on single parameters often need multiple base stations to coordinate location, such as the Chan algorithm[8] and the Caffery algorithm[9]. However, if multiple base stations are used, we must consider the synchronization between base stations, the pressure of signal transmission on the network, and the cost and management of hardware equipment. Also, to ensure the Quality of Service (QOS), the mobile terminal will only connect to the service base station in the wireless communication system, then multi-station cooperation will have the problem of whether the signal can be detected effectively at the same time[10, 11, 12]. Therefore, in many studies, the positioning algorithm based on multi-parameter fusion is used to reduce the number of cooperative cellular base stations[13], and it is best to use only one base station to operate. With the advance of the Multi Input Multi Output (MIMO) technology[14] and the smart antenna technology[15, 16], the realization of positioning technology based on parameter fusion becomes possible.

Compared with the multi-station, the single-station positioning algorithm has obvious advantages in equipment cost and complexity, response time, mobility, and flexibility. Therefore, the single station positioning system has high research value in the current era of advocating energy saving and high efficiency. The most common single-station positioning system relies on TOA/AOA hybrid positioning, as shown in Figure 1, which requires the existence of the Line-of-sight (LOS) path and sufficiently-accurate measurement parameters. However, in the outdoor environment with dense obstacles, the LOS path for the mobile terminal is often blocked, and the signal propagates through other paths to the base station in the form of the multi-path, which is called as the Non-line-of-sight (NLOS) propagation. If it is mistakenly recongnized as the LOS propagation, the accuracy of the positioning system will greatly deteriorate. Whether it is a single-station positioning system or a multistation positioning system, the influence of the NLOS propagation on positioning accuracy is poor. The positioning technology based on the multi-station mainly depends on suppressing the error caused by the NLOS and restoring LOS information, such as the classical Wylie algorithm[17]. In the single-station positioning system, the base station lacks other base stations to provide cooperative information, so it is difficult to conduct positioning under the condition of the NLOS, but combined with the advantages of the single station mentioned above, the research value of the single-station NLOS positioning technology is high.

The rest of this paper is organized as follow. The remainder of Section 1 give the previous studies on single station localization. Section 2 presents the positioning 
scheme, including the the description of the model, clustering algorithm, and target position calculation. Section 3 present the result of simulation, describing the influence of different factors on the positioning results, and use the the Wireless Insite to simulate the real environment to verify the positioning algorithm. Finally, Section 4 reports the conclusions.

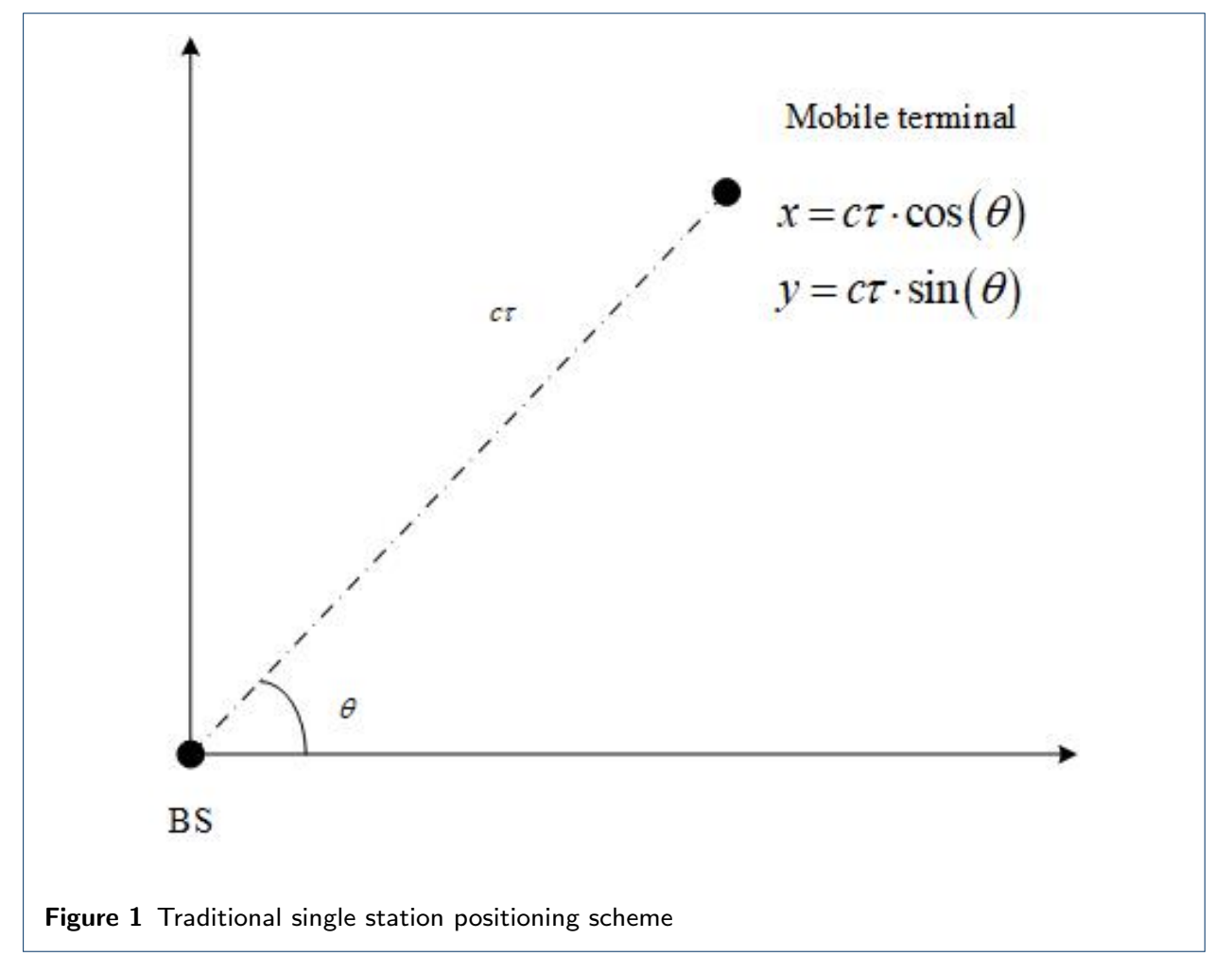

The single base station positioning system in the NLOS environment often has the problem of insufficient location information because of the lack of cooperation of other base stations, but correspondingly, the obstacles hindering the propagation of the LOS and the multipath signals generated by it can also provide information for positioning. By making full use of the information combined with mathematical statistics, machine learning and intelligent optimization can operate and improve localization accuracy[18].The authors in [19] use Support Vector Machine (SVM) to realize single station positioning in the MIMO system, but this scheme requires the Angel of Departure (AOD) of signal. For mobile terminals, it is difficult to measure this parameter. In the research of the single-site positioning technology in the NLOS environment, the idea of the research is to make use of the information of scatterers. A deterministic propagation model is used in [20]. In this scheme, the environmental information around the base station is sampled, the location of the scattering point is found according to the AOA of the multipath signal, and then the equation is established with the TOA to determine the location of the mobile terminal. However, this scheme requires $360^{\circ}$ omni-directional sampling of the environment near the base station. Therefore, the scatterer model is often introduced in the single base station localization system in the NLOS environment for analysis[21]. Common scatterer models include the Ring of Scattering (ROS), 
Disk of Scattering (DOS) and Gaussian Scattering Density Model (GSDM) [22, 23]. The authors in [24] quote ROS and DOS models and use the idea of reconstructing LOS paths to conduct positioning. This scheme analyzes the probability density function of TOA measurements of two models, takes the TOA of the LOS path as a parameter of the density function, and reconstructs the TOA of the LOS path by Bayesian estimation and maximum likelihood estimation. However, the algorithm of this scheme is complex, and the calculation process is also complicated. Based on the scattering point model, a pseudo-target dynamic feasible region constraint method is proposed in [25]. This algorithm extracts the AOA and the TOA of the multipath signal to recover multiple pseudo-targets, combines the scattering point model to determine the feasible region of the real target from the pseudo-target, and then estimates the position of the target. However, this scheme requires the maneuverability of the receiver and it is not suitable for base stations in cellular networks.

In order to solve the problems above, a scattering area model with the specific spatial layout information is proposed in this paper. First of all, in this model, the measurement parameters of the multipath signal are extracted by the base station, and then the parameters are clustered by the improved joint clustering algorithm. Second, the geometric equations are constructed according to the spatial layout information in the scattering area model and the system of equations established in this scheme can eliminate the clock synchronization error. Finally, the position of the target terminal is estimated by solving the equations by the Levenberg-Marquardt (LM) algorithm. This study is simulated by the Matlab and the Wireless Insite, and the simulation results show that the scheme can achieve outdoor NLOS single station station positioning and improve positioning accuracy.

\section{Methods}

\subsection{Scatterer model}

\subsubsection{Common scatterer model}

In previous researches, a variety of scatterer models have been proposed, among which the most commonly-used models are the ROS and the DOS, as shown in Figure 2. The ROS regards the scattering points to be randomly distributed on the circle with the target as the center and satisfies a certain numerical radius, and the DOS regards the scattering points as obeying the two-dimensional Gaussian random distribution in the circle. In the traditional scheme based on the ROS and the DOS, each time a multipath is introduced, the system of equations adds an unknown parameter, and too many unknown parameter will make the solving process of the system complicated.

\subsubsection{Scattering area model}

Aiming at the problem of the insufficient information in the traditional NLOS singlestation positioning system, this paper proposes a new signal reflection model in the NLOS environment. In the case of dense obstacles in the environment, there are often areas with reflected signals near the fixed base stations, such as tall or dense buildings. The propagation signal from the terminal is mainly reflected in these areas, and the scattering points are mainly distributed in these areas. In order 


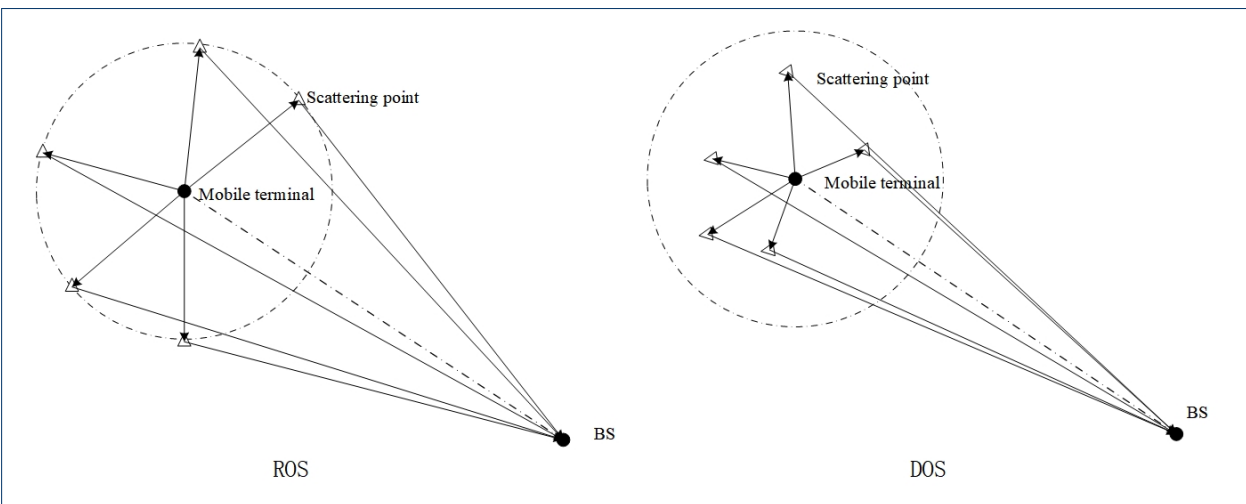

Figure 2 ROS and DOS

to facilitate analysis, the design of the scattering area model in this paper is as follows. Referring to the spatial layout of the environment near the base station, the scattering area is set as a circular area with a certain point as the center and a fixed radius. The center of the circle is regarded as the center of the scattering area and the scattering point is in the scattering area. The area obeys a two-dimensional Gaussian random distribution, and the average value is the center coordinate of the scattering area. The signal entering the scattering area is reflected by these scattering points and received by the base station. The scattering area model is shown in Figure 3.

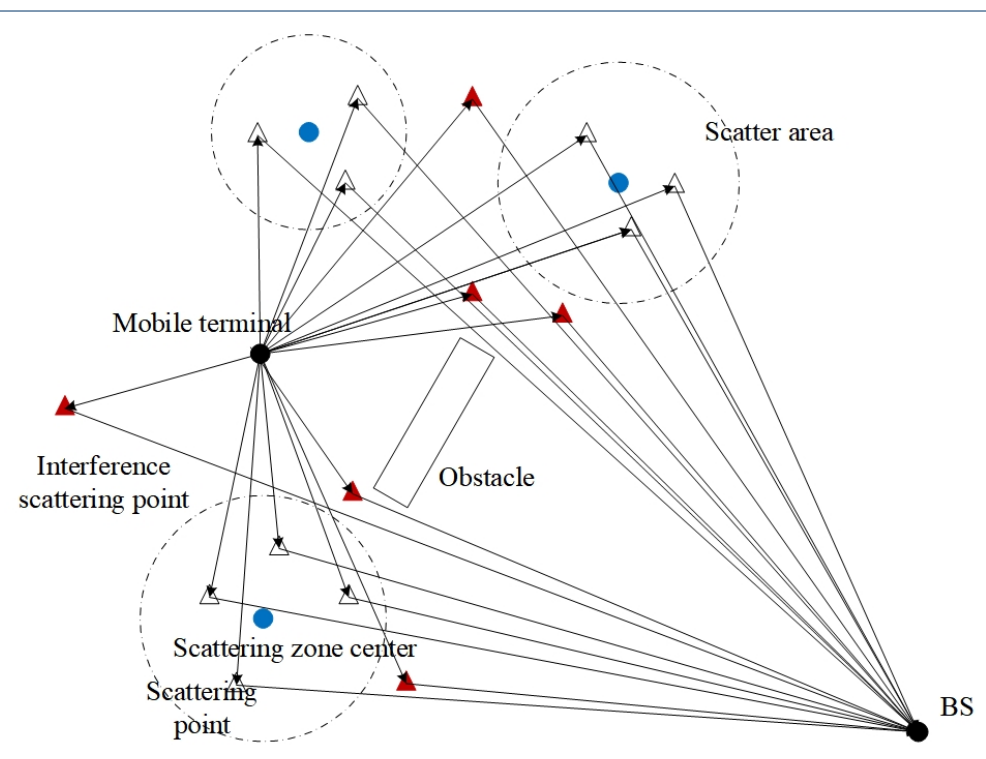

Figure 3 Scattering area model

The scatter area model is shown in Figure 3. Each blue solid circle is the center of a scattering area $\left(x_{i}, y_{i}\right), i=1,2, \ldots, N$, where $N$ is the number of scattering areas, and each dotted circle is a defined scattering area with the radius is $r_{i}$. The scattering point is in the circle, which obeys the two-dimensional Gaussian distribution with the mean value of the coordinate value of the scattering area 
center. Actually, scattering points that can reflect signals are not only limited in the defined scattering area but also exist far away from the scattering area. In this paper, they are called as interference scattering points, which are shown as red triangle points in Figure 3. The signal transmitted by the target is reflected by the scatter point and received by the base station. Letting $n$ be the number of measured multipaths, coordinates of scattering points are $\left(x_{s j}, y_{s j}\right), j=1,2, \ldots, n$, and $\left(x_{B}, y_{B}\right)$ is the coordinate of the base station.

\subsubsection{Measurement parameters}

In this scheme, the TOA and the AOA are extracted from the Channel State Information (CSI). Under the condition of the NLOS propagation, the TOA of the signal is the flight time that the signal is transmitted from the terminal to the scattering point, and then from the scattering point to the base station, the AOA is the relative angle between the scattering point of the scatter point and the base station. In the $4 \mathrm{G}$ or $5 \mathrm{G}$ wireless communication systems, the wireless signals relies on the Orthogonal Frequency Division Multiplexing (OFDM) technology to divide the system frequency band into several separate sub-carriers. The CSI on the subcarrier contains the relevant parameters of the carrier signal, and the information needed for positioning can be extracted by using the super-resolution parameter estimation technology. The scheme proposed in this paper works in the NLOS environment, that is, the environment with dense obstacles without the LOS.

The AOA and the TOA of the multipath signal arriving at the base station are represented as $\left(\theta_{j} . \tau_{j}\right), j=1,2, \ldots, n$, where $\theta_{J}$ is the AOA signal and $\tau_{j}$ is the TOA of the multipath, which are received at the base station. Generally, the propagation speed of the electromagnetic waves is constant, denoted as $c$, and then the relationship between the measured parameter and the scattering point is expressed as

$$
\left\{\begin{array}{l}
\theta_{j}=\arctan \left(\frac{y_{s j}-y_{B}}{x_{s j}-x_{B}}\right) \\
c \tau_{j}=c \tau^{\prime}{ }_{j}+\sqrt{\left(x_{s j}-x_{B}\right)^{2}+\left(y_{s j}-y_{B}\right)^{2}}
\end{array}\right.
$$

where $\tau_{j}^{\prime}$ is the time for the signal propagating from the target terminal to the $j$-th scattering point.

\subsection{Clustering algorithm}

According to the model constructed in this paper, the scattering points in the same scattering area have the characteristic of aggregation, by which the AOA and the the TOA have the similar distribution in the two-dimensional space, so the clustering algorithm can be used to process these parameters. The clustering algorithm is a type of unsupervised learning algorithms, which can be used to classify data without labels. We let $\left(\theta_{j} \cdot \tau_{j}\right)$ be the measurement parameter of the base station and the sample used for clustering be $S=\left\{s_{1}, s_{2}, \cdots, s_{n}\right\}$, where $s_{j}$ is the sample parameter, as shown in (2).

$$
s_{i}=\left\{\left(\alpha_{i}, \beta_{i}\right) \mid \alpha_{i}=c \tau_{i} \cdot \cos \left(\theta_{i}\right), \beta_{i}=c \tau_{i} \cdot \sin \left(\theta_{i}\right)\right\}
$$


Here, $s_{j}$ is also called as the pseudo target coordinate value determined by the $j$-th multipath measurement parameter, so each scattering point $\left(x_{s j}, y_{s j}\right)$ corresponds to a sample parameter $s_{j}$.

The scattering point $\left(x_{s j}^{i}, y_{s j}^{i}\right)$ in the scattering area $i$ is regarded as obeying the expected Gaussian distribution with the center of the scattering area $\left(x_{i}, y_{i}\right)$. If there are enough scattering points, the mean value of the scattering point coordinates in a scattering area approximately equals to the center of the scattering area $\left(x_{i}, y_{i}\right)$. Then the average value of the pseudo-target coordinate values corresponding to all the scattering points in the scattering area $i$ is approximately equal to the pseudotarget coordinate value formed by the signal reflected by the center of the scattering area $\left(x_{i}, y_{i}\right)$. If the average value after clustering is approximated as the pseudotarget coordinate value of the signal reflected from the center of the scattering area $\left(x_{i}, y_{i}\right)$, and the corresponding AOA and reach distance are calculated by using the average value of the clustering, a set of equations can be established to conduct positioning.

At present, there are many clustering algorithms in the existing research. Among them, the k-means clustering algorithm is based on the Euclidean distance between the data to calculate the similarity, so the operation is simple, and the time complexity tends to be linear in general[26]. However, the result of the k-means clustering algorithm is easily affected by the noise and the isolated sample points. The result of k-means clustering is shown in Figure 4. It can be seen that the outlier participating in clustering has a great influence on the clustering result. The above problem can be solved by using the mean shift clustering algorithm[27], which is based on the data distribution density to measure the similarity. The interference points are scattered and deviate from the main scattering area. The algorithm will separate them into clusters. However, the sliding window size of mean shift clustering has an important influence on clustering performance. In this paper, the window size is related to the radius of the scattering area. If all data points are clustered once, the window size of mean shift clustering should be set based on the maximum scattering area, but the clustering performance of the clusters corresponding to the scattering area with a smaller radius is poor. The results of mean shift clustering are shown in Figure 5, in which the dashed circle represents the size of the clustering window. The fixed-size sliding window does not have a good clustering effect when the difference in the radius of the scattering area is too large, and some points are even not classified into the cluster.

This paper combines two clustering algorithms to process parameters. First of all, we use k-means clustering to roughly distinguish all data and then locally use mean shift clustering to filter out interference points on the result of $\mathrm{k}$-means clustering.

The cluster center after k-means clustering on all data is $\left(\alpha^{\prime}{ }_{i}, \beta^{\prime}{ }_{i}\right)$. Each result corresponds to a scattering area, and the center of the scattering area is $\left(x_{i}, y_{i}\right)$, and with the corresponding radius $r_{i}$. For each k-means clustering result, we calculate the window radius of mean shift clustering by the following equaltion.

$$
w_{i}=r_{i} \cdot \frac{2 \sqrt{\left(\bar{\alpha}_{i}-x_{B}\right)^{2}+\left(\bar{\beta}_{i}-y_{B}\right)^{2}}}{\sqrt{\left(x_{i}-x_{B}\right)^{2}+\left(y_{i}-y_{B}\right)^{2}}}
$$



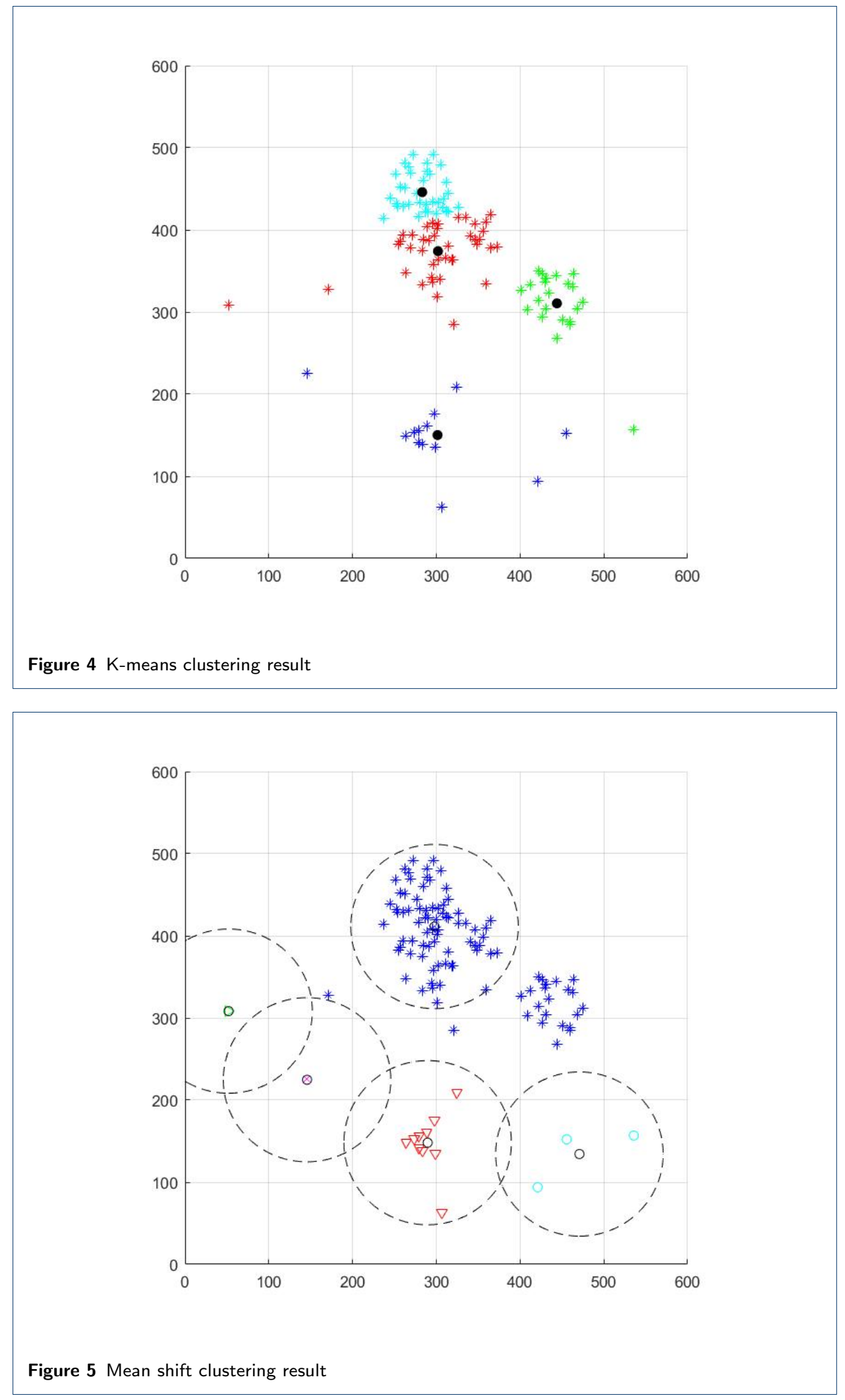

The clustering result of the joint clustering algorithm is shown in Figure 6. The size of the dotted circle represents the sliding window size of the mean shift cluster 
in each k-means clustering result, and each dotted circle represents a cluster. In Figure 6 the clusters with a large number of distributed points represent the clustering results of the pseudo-target corresponding to the reflection signals of the scattered points in the scattering area, and the clusters with fewer distributed points represent the clustering result of the pseudo-target corresponding to the interference scattering points. The clustering results with the largest number of pseudo-target points are selected as references, and the clustering center is used as a parameter to establish an equation set, whereas other clustering results are discarded as the corresponding results of interference scattering points.

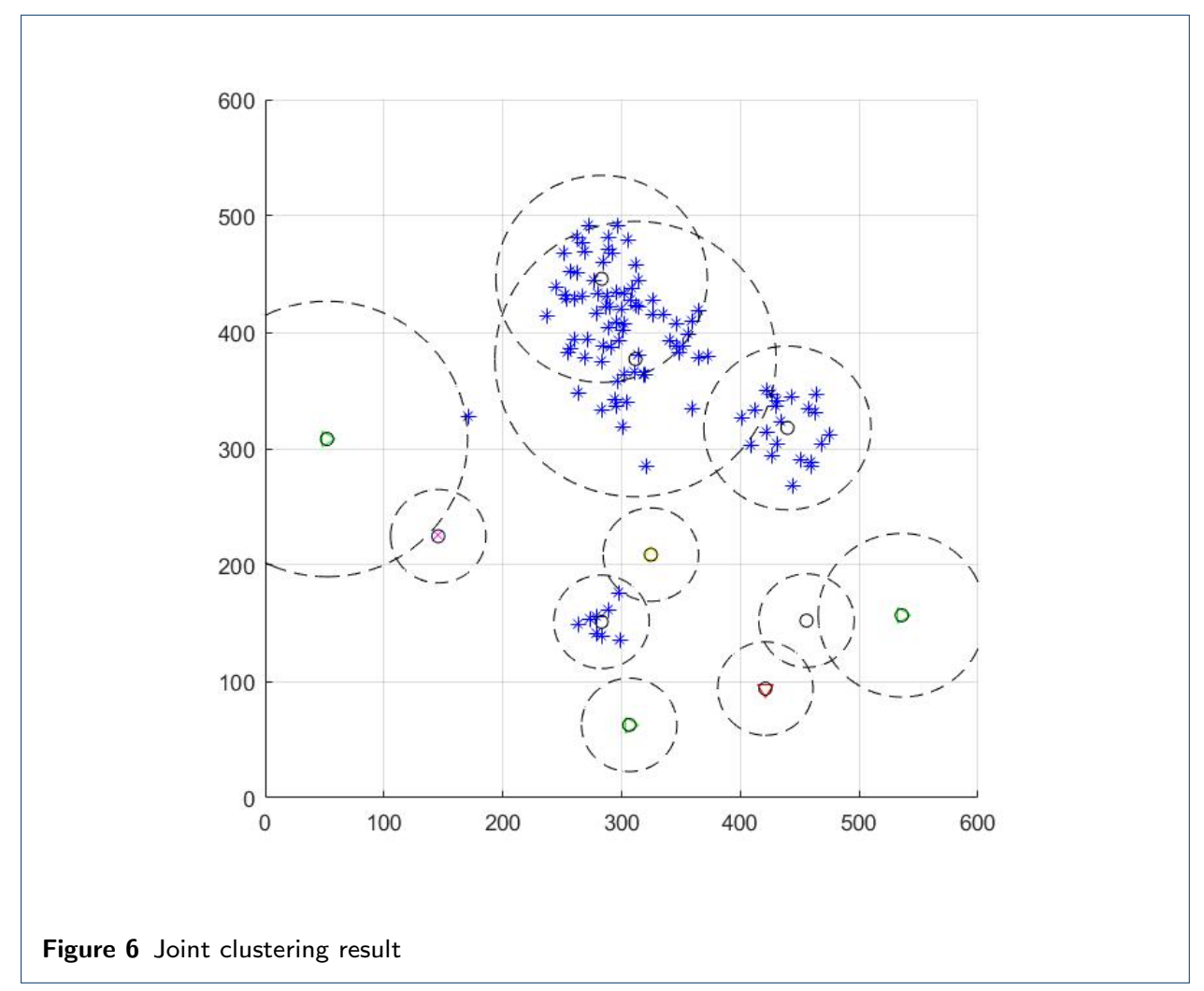

\subsection{Target position calculation}

The information that we have got involve the center position of scattering areas $\left(x_{i}, y_{i}\right), i=0,1, \ldots, N$ and the clustering result obtained by the joint clustering algorithm $\left(\bar{\alpha}_{i}, \bar{\beta}_{i}\right)$. The corresponding parameter $\left(\bar{\theta}_{i}, \bar{\tau}_{i}\right)$ is deduced from (1), and then the AOA between the center of the scattering area $\left(x_{i}, y_{i}\right)$ and the base station is calculated as

$$
\theta_{i}^{\prime}=\arctan \left(\frac{y_{i}}{x_{i}}\right) \quad i=0,1, \ldots, N
$$

The parameters $\bar{\theta}_{i}$ and $\theta_{i}^{\prime}$ are matched according to the principle of the minimum difference, and $\left(\bar{\theta}_{i}, \bar{\tau}_{i}\right)$ is taken as the approximate measurement parameter with the scattering center $\left(x_{i}, y_{i}\right)$. If the coordinate of the target terminal is $(x, y)$, the 


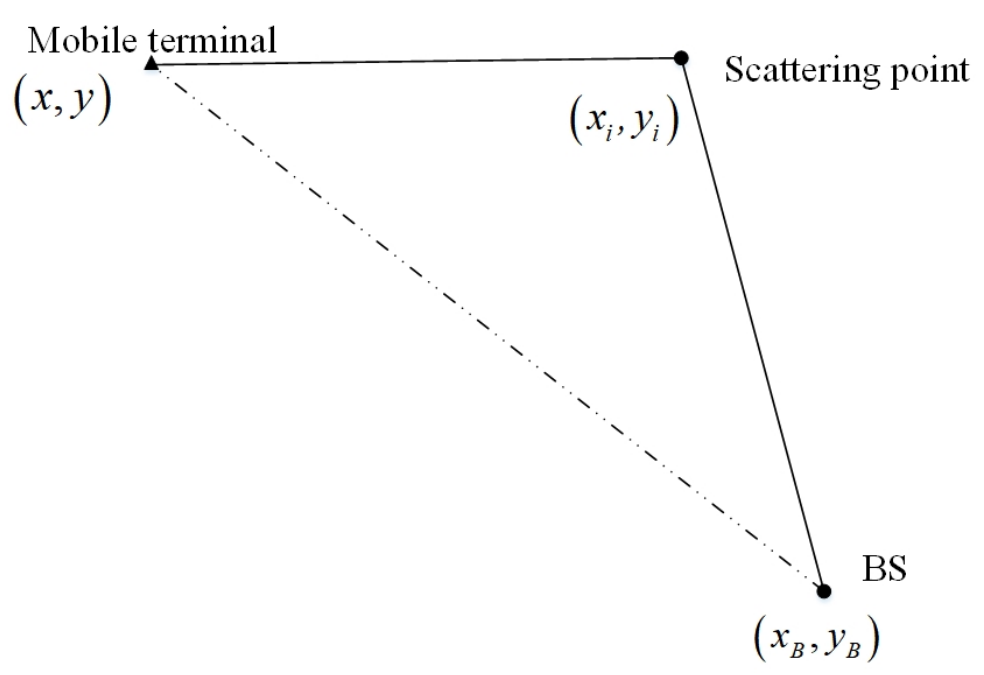

Figure 7 Single station positioning structure in the NLOS

relations of the target, the scattering point, and the base station are shown in Figure 7.

According to the geometric structure in Figure 7, we can establish

$$
\left\{\begin{array}{c}
\sqrt{\left(x-x_{1}\right)^{2}+\left(y-y_{1}\right)^{2}}=c \bar{\tau}_{1}-\sqrt{\left(x_{1}-x_{B}\right)^{2}+\left(y_{1}-y_{B}\right)^{2}} \\
\sqrt{\left(x-x_{2}\right)^{2}+\left(y-y_{2}\right)^{2}}=c \bar{\tau}_{2}-\sqrt{\left(x_{2}-x_{B}\right)^{2}+\left(y_{2}-y_{B}\right)^{2}} \\
\vdots \\
\sqrt{\left(x-x_{N}\right)^{2}+\left(y-y_{N}\right)^{2}}=c \bar{\tau}_{N}-\sqrt{\left(x_{N}-x_{B}\right)^{2}+\left(y_{N}-y_{B}\right)^{2}}
\end{array}\right.
$$

In the actual communication process between the target terminal and the base station, the parameters have the synchronization error due to the out of sync of clocks between the transmitter and the receiver, that is

$$
\tau_{T O A}=\tau_{\text {TOA_true }}+\tau_{s_{-} \text {err }}+\tau_{n}
$$

where $\tau_{\text {TOA_true }}$ is the true TOA, $\tau_{s_{-} e r r}$ is the delay of the synchronization error, and $\tau_{n}$ is the measurement error caused by the white noise. In the single station positioning system, the clock synchronization error of each multipath is the same in the same signal transmission process, so the difference equation of two multipath TOAs is established to eliminate the synchronization error. By assuming that the distance from the target to the scattering point is $l_{i}(i=0,1, \cdots, N)$, we have

$$
l_{i}=c \bar{\tau}_{i}-\sqrt{\left(x_{i}-x_{B}\right)^{2}+\left(y_{i}-y_{B}\right)^{2}}
$$


If the number of scattering areas is $N$, the $N-1$ equations can be established as

$$
\left\{\begin{array}{c}
\sqrt{\left(x-x_{N}\right)^{2}+\left(y-y_{N}\right)^{2}}-\sqrt{\left(x-x_{1}\right)^{2}+\left(y-y_{1}\right)^{2}}=l_{N}-l_{1} \\
\sqrt{\left(x-x_{N}\right)^{2}+\left(y-y_{N}\right)^{2}}-\sqrt{\left(x-x_{2}\right)^{2}+\left(y-y_{2}\right)^{2}}=l_{N}-l_{2} \\
\vdots \\
\sqrt{\left(x-x_{N}\right)^{2}+\left(y-y_{N}\right)^{2}}-\sqrt{\left(x-x_{N-1}\right)^{2}+\left(y-y_{N-1}\right)^{2}}=l_{N}-l_{N-1}
\end{array}\right.
$$

In practice, the (8) is not exactly equal. The solving of deterministic equation can be transformed into the solving of optimization problem. First, we rewrite (8) as

$$
\begin{aligned}
\varphi_{i}(x, y) & =\sqrt{\left(x-x_{N}\right)^{2}+\left(y-y_{N}\right)^{2}}-\sqrt{\left(x-x_{i}\right)^{2}+\left(y-y_{i}\right)^{2}}-\left(l_{N}-l_{i}\right), \\
i & =0,1, \cdots, N-1
\end{aligned}
$$

where $\varphi_{i}(x, y)$ is the error objective function. Then, The solution that minimizes the objective function shown in (10) is the position of the target.

$$
\varepsilon(x, y)=\frac{1}{2} \sum_{i=1}^{N-1} \varphi_{i}^{2}(x, y)
$$

This paper uses the the LM algorithm to solve (10). The LM algorithm is an iterative algorithm for finding the extreme value of a function, which can be used to solve the nonlinear least squares problem. The positioning scheme in this paper introduces the spatial layout as an information supplement and uses a clustering algorithm to process multipath parameters, which can greatly simplify equations and avoid the situation that the function does not converge in the limited domain. In the LM algorithm, the solution that minimizes the objective error function is the optimal solution, which is the estimation of the target terminal position.

\section{Results and discussion}

The positioning range is $300 \mathrm{~m}$ by $300 \mathrm{~m}$, and the coordinate of the base station is $(0,0)$. The parameters of the AOA and the TOA are generated by the simulation of the scattering area model. With the known central coordinates and radius of the scattering area, a certain number of random points are distributed in each scattering area. These random points obey the two-dimensional Gaussian distribution with the mean value of the coordinate value of the scattering area center, and the random points within the radius of the scattering area are selected as scattering points. In addition, in order to test the performance of interference scattering points outside the scattering area, several interference scattering points are randomly generated in the positioning range. The TOA is the sum of the flight time of the signal from the mobile terminal to the scattering point and the flight time from the scattering point to the base station, and the flight distance of the signal is the TOA multiplied by $c$. The relative angle between scattering points and the base station represents the simulated AOA. 200 independent experiments are conducted in each round of simulation experiments. 


\subsection{Influence of Model Variables}

Figure 8 shows the effect of the number and size of scattering areas on the result. All scattering areas are set with the same radius during the simulation. It can be seen from the figure that when the number of scattering areas is less than 5 , the positioning accuracy increases with the increase of the number of scattering areas. However, when the number of scattering areas increase to 6 , the positioning accuracy is decreases. Too many scattering areas and scattering points lose their clustering characteristics, resulting in a decrease in clustering effect. When the radius is less than $20 \mathrm{~m}$, the larger the radius of the scattering area, the smaller the positioning error. When the radius is greater than $20 \mathrm{~m}$, the scattering points will lose the clustering characteristics, resulting in a decrease in the clustering effect.

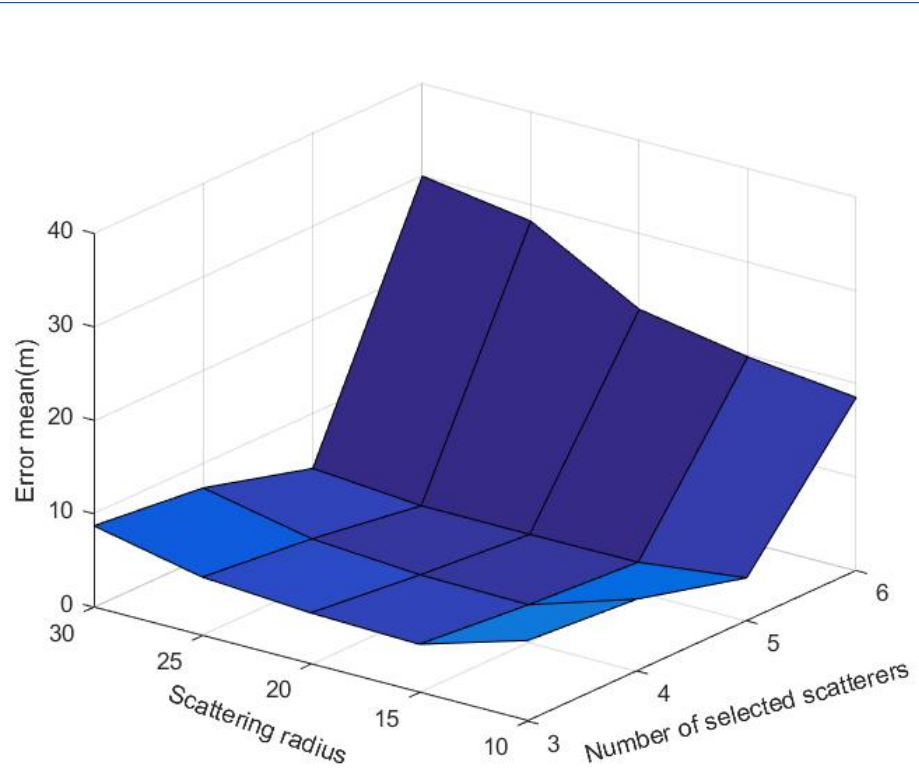

Figure 8 Influence of the number and size of scattering areas on positioning performance

It can be seen from the above analysis that the structure of the scattering area has an important influence on the positioning result. The method of determining the scattering area is as follows. We examine the spatial layout of the main reflection signal area near the base station, such as the location of the building group, initially determine the location of the scattering area, and then combine the multipath signal parameters measured by the base station to determine the specific scattering area. After that, we optimize each area into a circular scattering area and use the center of the circle as the center of the scattering area. By considering the environment, when selecting the scattering area, it should be ensured that the scattering area covers a wide area, and there is still enough space between the scattering areas.

Figure 9 shows the effect of the number of random points and interference scattering points on positioning accuracy. The number of multipaths equals to the number of scattering points. In the simulation process, the more random points preset for the scattering area, the more scattering points are distributed within the radius of the scattering area. Obviously, the more random points, that is, the more multipaths extracted by the base station, the higher the positioning accuracy. When no 
interference point is introduced, the sample parameters are corresponding to the scattering points in the scattering area, and the results after clustering the sample parameters correspond to the respective scattering areas, the parameter $\left(\bar{\theta}_{i}, \bar{\tau}_{i}\right)$ calculated by the clustering center is approximately estimated as the parameter taking the center of the scattering area $\left(x_{i}, y_{i}\right)$ as the scattering point. Thus, when there is no interference scattering point, the positioning result has the error, but the error is small, generally within $5 \mathrm{~m}$. As the number of interference scattering points increases, the mean value and mean square error of the error show an upward trend. However, when the number of random points pre-allocated more than 80 , the number of interference points has little effect on the mean error. Therefore, the joint clustering algorithm can effectively reduce the influence of interference scattering points on positioning accuracy when enough multipaths are extracted.

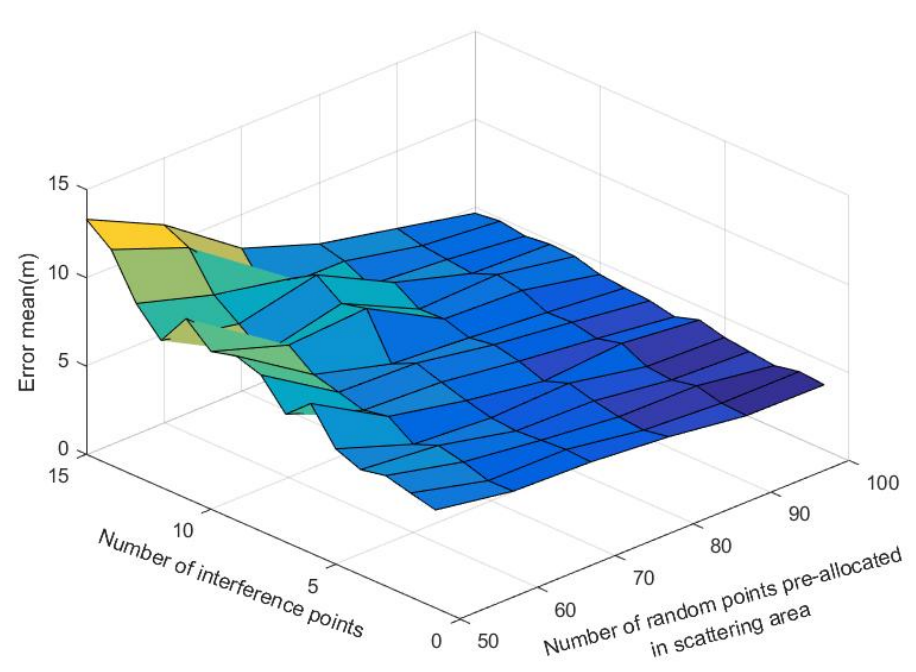

Figure 9 Influence of interference points and pre-assigned random points on the result

In the process of simulation, the white noise error is added to the AOA and the TOA, the mean value of the noise is 0 , and the noise intensity is measured by the standard deviation of the error. Since the propagation velocity of electromagnetic wave is always $c$, the TOA error can be measured directly by the measured flight distance error. Figure 10 shows the influence of the measurement parameter error on positioning accuracy, from which we can find the greater the noise standard deviation, the greater parameter accuracy affected by the noise.

\subsection{Influence of Clustering}

K-means clustering, mean shift clustering, and joint clustering algorithms are used to cluster the parameters and realize positioning. The simulation results are shown in Figure 11. In the fixed scene, the positioning result of the mean shift clustering algorithm is better than that of the k-means clustering algorithm, and the positioning result of the joint clustering algorithm is slightly better than using the mean shift clustering algorithm. 


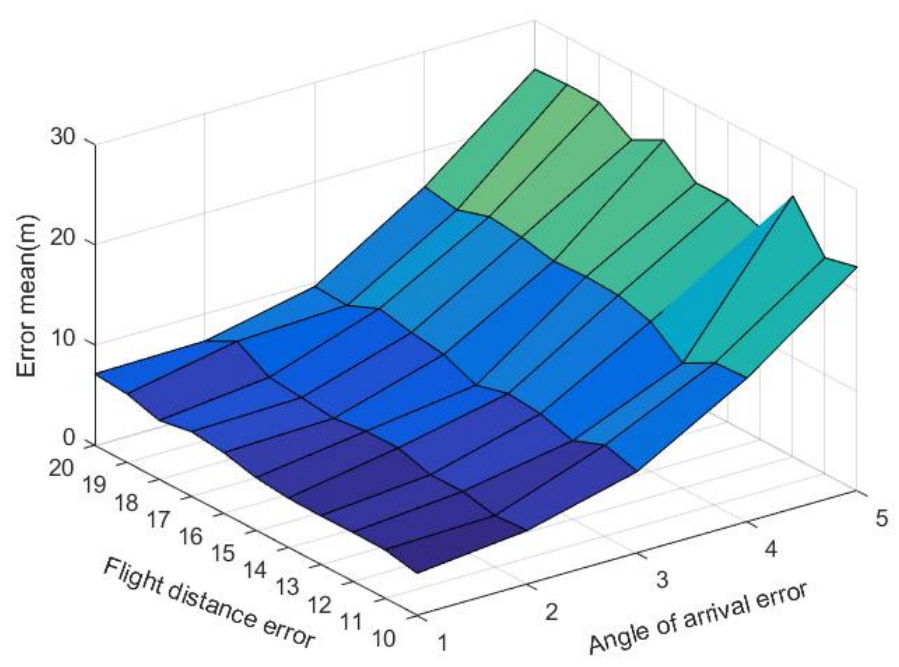

Figure 10 Influence of parameter measurement error on the result

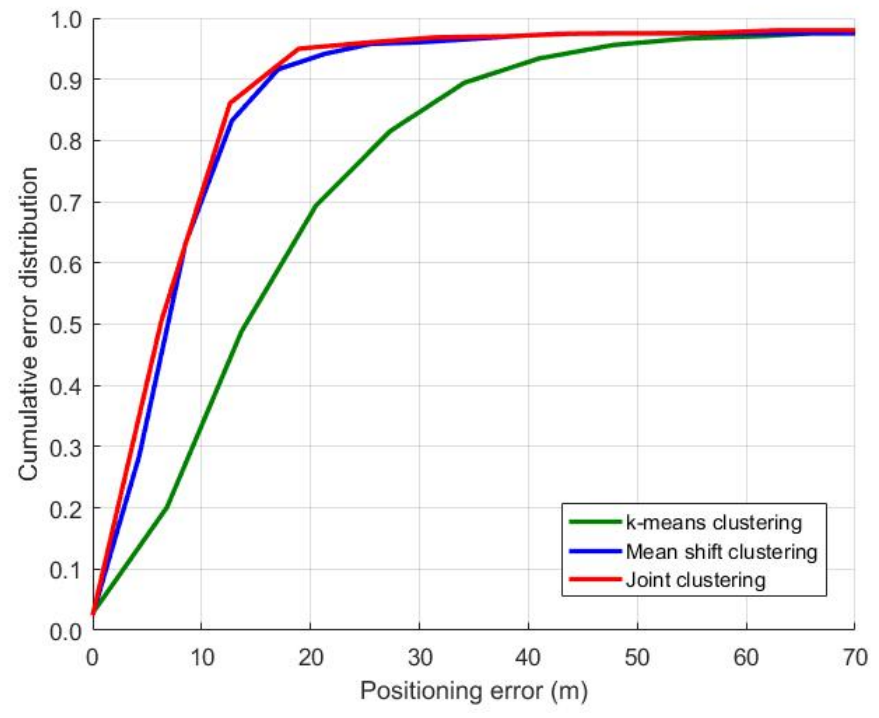

Figure 11 Result by different clustering algorithms

According to the previous analysis, compared to using mean shift clustering, the advantage of joint clustering is that it is applicable to a variety of radius sizes of the scattering area. In order to verify this feature of joint clustering, when comparing the positioning results of using mean shift clustering and using joint clustering,the radius of each scattering area takes a random value in $(0,40]$, and we repeat 200 independent experiments. The simulation results are shown in Figure 12. At this time, the positioning effect based on joint clustering is better than the positioning effect based on mean shift clustering. Since the joint clustering algorithm still has 
a good clustering result in the case of great difference in the radius of the scattering area. Therefore, the joint clustering algorithm is more suitable for the model proposed in this paper.

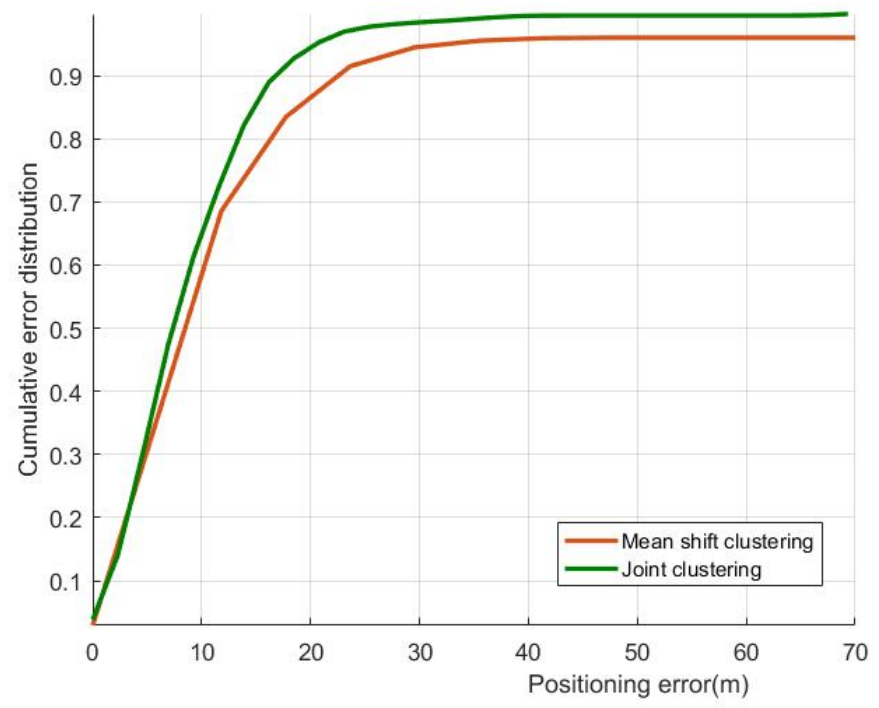

Figure 12 Error CDF based on joint clustering and mean shift clustering when the scattering radius is set randomly.

\subsection{Comparison of Different Schemes}

The result of the proposed scheme is compared with the ones by using ROS and DOS models, and the results are shown in Figure 13. The positioning effect of the model scheme in this paper is better than both the ROS model and the DOS model. The positioning error based on the ROS model is smaller than that based on the DOS model, the setting of the scattering points of the ROS model on the ring can not be fully verified in reality, and it is difficult to determine the radius of the ring in the complex environment. Compared with the ROS model, the DOS model is more appropriate to describe the complex environment, but compared with the scheme of this paper, the introduction of the spatial layout information is still insufficient. The simulation results show that the positioning result of the scheme in this paper is obviously better compared to the DOS model.

\subsection{Wireless Insite Simulation Data}

Wireless Insite is a set of simulation software using the Ray Tracing (RT) model, which can be used to analyze the working characteristics of radio wave transmission and wireless communication system in individual scenes. It can simulate and predict the electromagnetic wave transmission path and the working characteristics of each channel of the communication system in the complex large geographical areas, urban areas, relatively small indoor environments, and mixed environments. In this paper we use the Wireless Insite software to simulate the actual environment and generate the corresponding data to verify the scheme of this paper. 


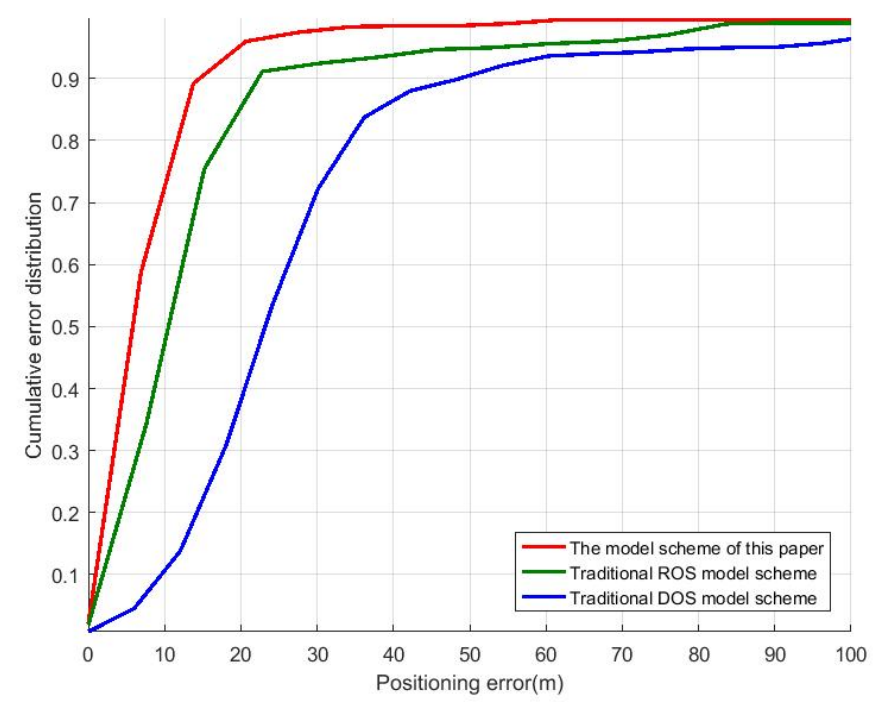

Figure 13 Error CDF of different schemes

First of all, the actual scene model is constructed by using the Wireless Insite, based on which the scene environment between the Digital Library in the Chongqing University of Posts and Telecommunications (CQUPT) and the Xinke Building is constructed. As shown in Figure 14 and Figure 15, the building in the picture is set according to the building structure in the actual scene, the model material is set as the cement concrete, the base station is arranged according to the actual planning, and the yellow dot in Figure 15 is the location of the base station (only one base station is used at a time). The position of the blue point is the position set by the terminal target, and these positions are consistent with the NLOS condition in the process of simulation.

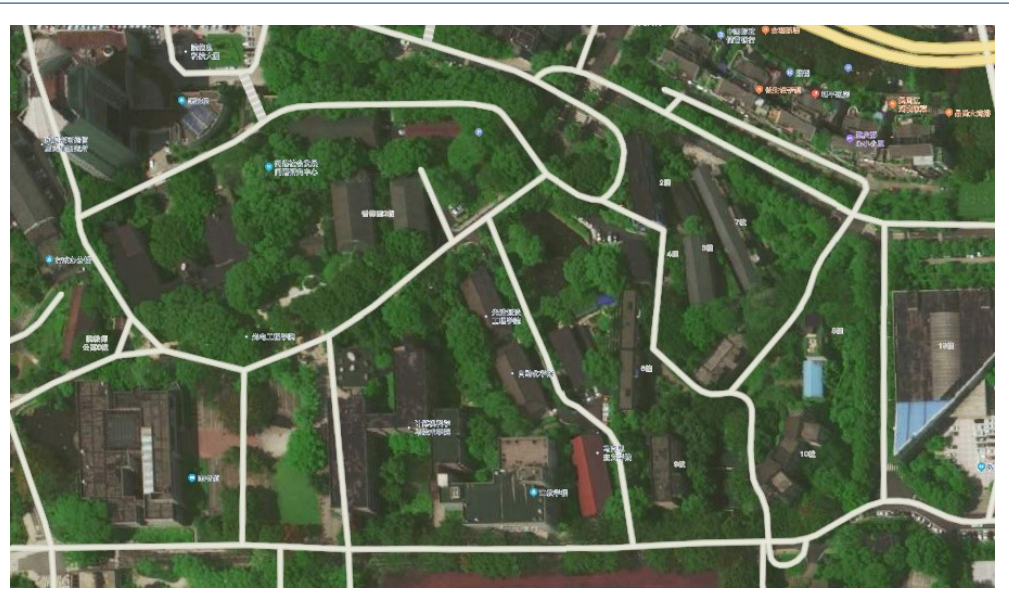

Figure 14 Satellite scan image of simulation scene 


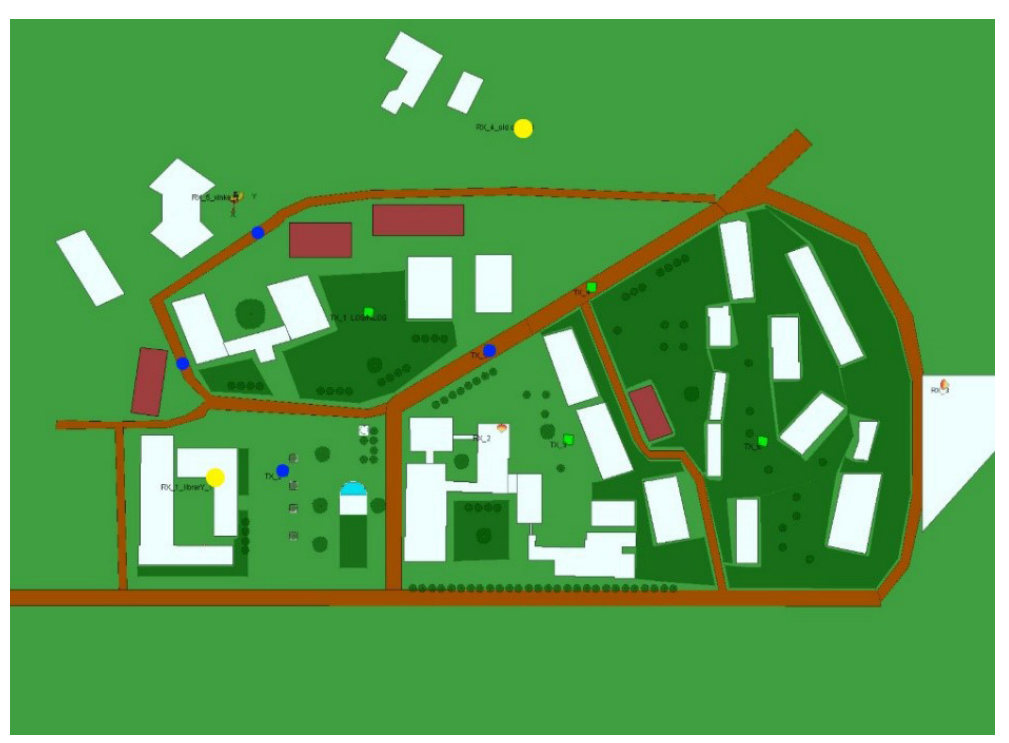

Figure 15 Map model built by the Wireless Insite

By taking the target location downstairs of the Xinke Building and the base station on the library in the CQUPT as an example, the simulation ray is shown in Figure 16.

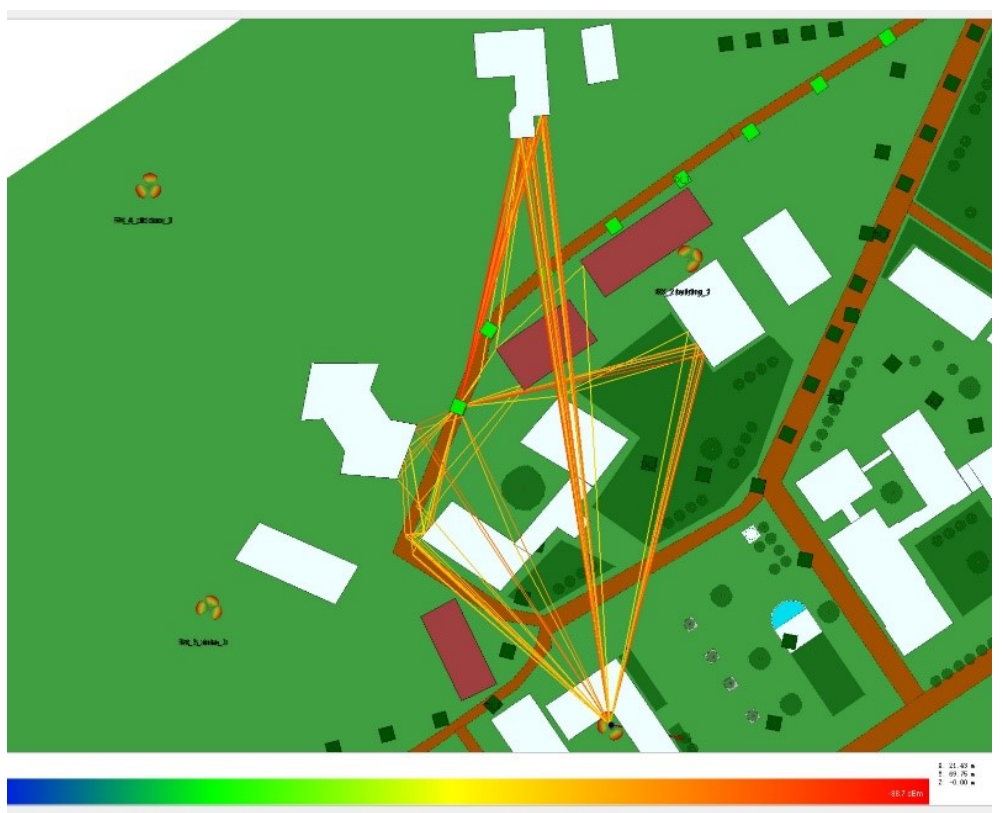

Figure 16 Simulation ray by the Wireless Insite

According to the environmental layout characteristics of the scene area and the relevant information of signal rays, the scattering area is determined. As can be seen from Figure 16, in this environment, the scattering area mainly depends on the selection of the location of the building. The green square in the figure represents 
an area where the terminal is located with the range of $5 \mathrm{~m}$ by $5 \mathrm{~m}$, a sampling point is taken with the interval of $1 \mathrm{~m}$, and 25 sampling points are taken for simulation verification, as shown in Figure 17.

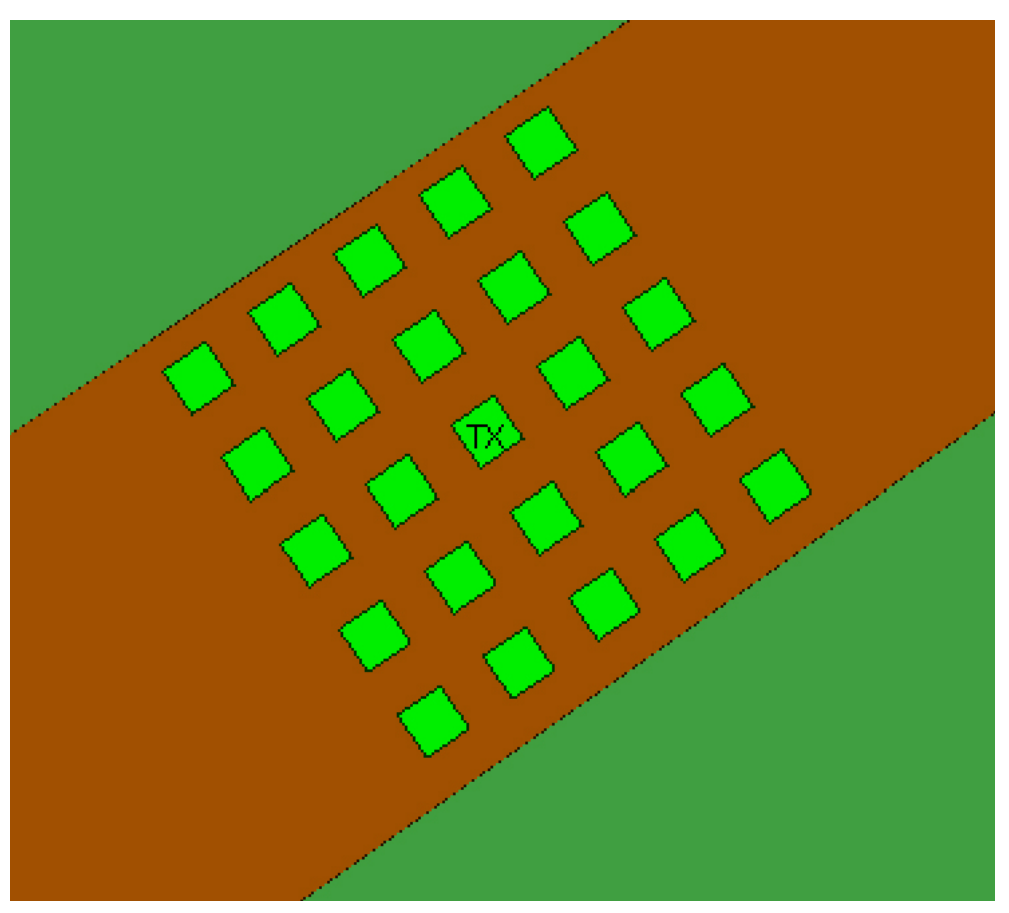

Figure 17 Schematic diagram of sampling points

Multiple regions are sampled, the error results are counted, and the Cumulative Distribution Function (CDF) diagram is shown in Figure 18. In this scheme, 65\% of the error obtained by using the simulation data of Wireless Insite software can be controlled within $21 \mathrm{~m}$.

\section{Conclusion}

This paper proposes a novel scattering area model in the actual scene. Compared with the traditional ROS and DOS models, the proposed model performs better when they are used for single base station positioning in the outdoor NLOS environment. Based on the proposed scattering area model, this paper combines k-means clustering and mean shift clustering algorithms to deal with measurement parameters, for the sake of achieving the good clustering performance. After performing clustering on these parameters, we combine the sample parameters and the spatial layout to establish the equation group, which contributes to eliminating the synchronization error. Finally, the solving of the equation group is converted into the solving of the least squares optimization problem, and the location of the target is calculated by the LM algorithm. Verified by simulation, the proposed scheme can achieve high positioning accuracy under the condition of the outdoor NLOS with a single base station. In addition, the algorithm proposed makes full use of the environmental information and parameters of the multipath signal for positioning. To summarize, the proposed system is feature with high positioning accuracy and low complexity. 


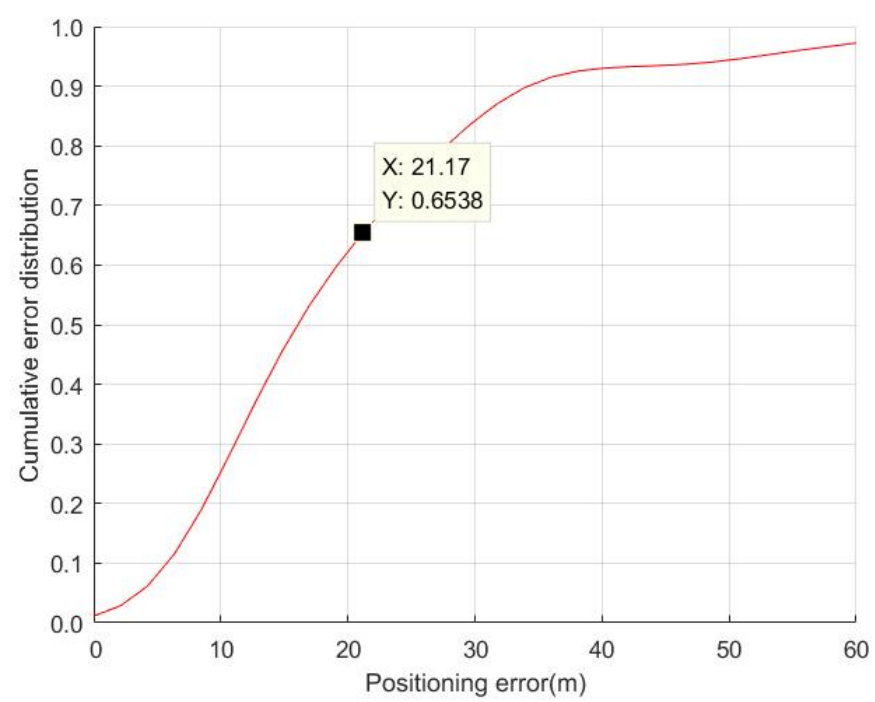

Figure 18 Wireless Insite simulation results

\section{Acknowledgements}

This work was supported by the School of Communication and Information Engineering, Chongqing University of Posts and Telecommunications.

Funding

This work was supported in part by the Science and Technology Research Program of Chongqing Municipal Education Commission (KJZD-K202000605, KJQN202000630, KJQN201900603), the Chongqing Natural Science Foundation Project (cstc2020jcyj-msxmX0842, cstc2020jcyj-msxmX0865), and the National Natural Science Foundation of China (61901076).

Abbreviations

GPS: Global Positioning System; BDS: BeiDou Navigation Satellite System; FCC: Federal Communications Commission; E911: Enhanced 911; LBS: Location Based Service; TOA: Time of Arrival; TOF: Time of Arrival; AOA: Angle of Arrival; TDOA: Time Difference of Arrival; RSS: Received Signal Strength; QOS: Quality of Service; MIMO: Multi Input Multi Output; LOS: Line-of-sight; NLOS: Non-line-of-sight; SVM: Support Vector Machine; AOD: Angel of Departure; ROS: Ring of Scattering; DOS: Disk of Scattering; GSDM: Gaussian Scattering Density Model; LM: Levenberg-Marquardt; OFDM: Orthogonal Frequency Division Multiplexing; RT: Ray Tracing; CQUPT: Chongqing University of posts and Telecommunications; CDF: Cumulative Distribution Function

Availability of data and materials

All data generated or analyzed during this study are included in this paper.

Ethics approval and consent to participate

This paper does not contain any studies with human participants or animals performed. All data and procedures performed in this paper were in accordance with the ethical standards of research community.

Competing interests

The authors declare that they have no competing interests.

Consent for publication

Not applicable.

Authors' contributions

The algorithms proposed in this paper have been conceived by Y. Wang, Q. Wu, and M. Zhou. Y. Wang, Q. Wu and $\mathrm{X}$. Yang designed and performed the experiments and wrote the paper. M. Zhou, W. Nie and L. Xie investigated, validated and revised this paper. The authors approved the final manuscript.

Authors' information

Yong Wang, School of Communication and Information Engineering, Chongqing University of Posts and Telecommunications, Chongqing, China.

Qihong Wu, School of Communication and Information Engineering, Chongqing University of Posts and Telecommunications, Chongqing, China.

Mu Zhou, School of Communication and Information Engineering, Chongqing University of Posts and

Telecommunications, Chongqing, China. 
Xiaolong Yang, School of Communication and Information Engineering, Chongqing University of Posts and Telecommunications, Chongqing, China.

Wei Nie, School of Communication and Information Engineering, Chongqing University of Posts and Telecommunications, Chongqing, China.

Liangbo Xie, School of Communication and Information Engineering, Chongqing University of Posts and Telecommunications, Chongqing, China.

Author details

School of Communication and Information Engineering, Chongqing University of Posts and Telecommunications, Chongqing, China.

\section{References}

1. A. Blanco, N. Ludant, P. J. Mateo, Z. Shi, Y. Wang, J. Widmer.: Performance Evaluation of Single Base Station ToA-AoA Localization in an LTE Testbed. 2019 IEEE 30th Annual International Symposium on Personal, Indoor and Mobile Radio Communications (PIMRC), pp. 1-6 (2019)

2. K. Kitao, T. Imai, M. Inomata, and et al.: Study on Path Identification at $40 \mathrm{GHz}$ Band in NLOS Urban Street Cell Environment. 2019 IEEE Wireless Communications and Networking Conference (WCNC), pp. 1-4 (2019)

3. M. Zhou, Y. Wang, Z. Tian, Y. Lian, Y. Wang, B. Wang.: Calibrated data simplification for energy-efficient location sensing in internet of things. IEEE Internet of Things Journal. 6(4), 6125-6133 (2019)

4. M. Zhou, Y. Wang, Y. Liu, Z. Tian.: An information-theoretic view of WLAN localization error bound in GPS-denied environment. IEEE Transactions on Vehicular Technology. 68(4): 4089-4093 (2019)

5. Federal C. C.: Revision of the commission's rules to ensure compatibility with enhanced 911 emergency calling systems. Report and Order and Further Notice of Proposed Rulemaking, Tech. Rep. CC Docket. 94-102 (1996)

6. N. Garcia, H. Wymeersch, E. G. Larsson, A. M. Haimovich and M. Coulon.: Direct Localization for Massive MIMO. IEEE Transactions on Signal Processing. 65, 2475-2487 (2017)

7. S. Wu, S. Zhang, D. Huang.: A TOA-Based Localization Algorithm With Simultaneous NLOS Mitigation and Synchronization Error Elimination. IEEE Sensors Letters. 3, 1-4 (2019)

8. Chan Y T, Ho K C.: A simple and efficient estimator for hyperbolic location. IEEE Transactions on signal processing. 42(8), 1905-1915 (1994)

9. Caffery J J.: A new approach to the geometry of TOA location. IEEE Vehicular Technology Conference. Boston: IEEE Press, pp. $1943-1949$ (2000)

10. C. Li, S. Zhang, P. Liu, F. Sun, J. M. Cioffi, L. Yang.: Overhearing protocol design exploiting inter-cell interference in cooperative green networks. IEEE Transactions Vehicular Technology. 65, 441-446 (2016)

11. C. Li, P. Liu, C. Zou, F. Sun, J. M. Cioffi, L. Yang.: Spectral-efficient cellular communications with coexistent one- and two-hop transmissions. IEEE Transactions on Vehicular Technology, vol. 65, 6765-6772 (2016)

12. J. Su, R. Xu, S. Yu, B. Wang, J. Wang.: Redundant rule detection for software-defined networking. KSII Transactions on Internet and Information Systems. 14, 2735-2751 (2020)

13. M. Zhou, X. Li, Y. Wang, S. Li, Y. Ding, W. Nie.: 6G multi-source information fusion based indoor positioning via Gaussian kernel density estimation. IEEE Internet of Things Journal. (2021)

14. C. Li, F. Sun, J. M. Cioffi, L. Yang.: Energy efficient MIMO relay transmissions via joint power allocations. IEEE Transactions on Circuits and Systems. 61, 531-535 (2014)

15. C. Li, H. J. Yang, F. Sun, J. M. Cioffi, L. Yang.: Multiuser overhearing for cooperative two-way multiantenna relays. IEEE Transactions Vehicular Technology. 65, 3796-3802 (2016)

16. C. Li, H. J. Yang, F. Sun, J. M. Cioffi, L. Yang. Cioffi.: Adaptive overhearing in two-way multi-antenna relay channels. IEEE Signal Processing Letters. 23, 117-120 (2016)

17. M. P. Wylie, J. Holtzman.: The non-line of sight problem in mobile location estimation. Proceedings of ICUPC - 5th International Conference on Universal Personal Communications, Cambridge, MA, USA, 1996, pp. 827-831 (1996)

18. J. Su, R. Xu, S. Yu, B. Wang, J. Wang.: Idle slots skipped mechanism based tag identification algorithm with enhanced collision detection. KSII Transactions on Internet and Information Systems. 14, 2294-2309 (2020)

19. Jianping Zhang, Yongning Zhuo, Yi Zhao.: Mobile location based on SVM in MIMO communication systems. 2010 International Conference on Information, Networking and Automation (ICINA), Kunming, 2010, pp.360-363 (2010)

20. M. Porretta, P. Nepa, G. Manara, F. Giannetti, M. Dohler, B. Allen, A.H. Aghvami.: A novel single base station location technique for microcellular wireless networks: description and validation by a deterministic propagation model. IEEE Transactions on Vehicular Technology. 53, 1502-1514 (2004)

21. Zhaounia M, Landolsi M A, Bouallegue R.: Mobile localization under non-line-of-sight conditions using scattering information. International Journal of Wireless Information Networks. 17(1-2), 1-10 (2010)

22. S. Al-Jazzar, J. Caffery, H. You.: Scattering-model-based methods for TOA location in NLOS environments. IEEE Transactions on Vehicular Technology. 56, 583-593 (2007)

23. A. Borhani, M. Patzold.: A unified disk scattering model and its angle-of-departure and time-of-arrival statistics. IEEE transactions on vehicular technology. 62, 473-485 (2013)

24. S. Al-Jazzar, J. Caffery.: ML and Bayesian TOA location estimators for NLOS environments. Proceedings IEEE 56th Vehicular Technology Conference, Vancouver, BC, Canada, 2002, pp. 1178-1181 (2002)

25. G. Shao.: Research on the positioning algorithm under single base station in NLOS environment. Lanzhou University of Technology (2014)

26. S. T. Yoon, K. S. Shim.: K-means clustering for handling high-dimensional large data. Journal of KIISE: Computing Practices and Letters. 18, 55-59 (2012)

27. Leichter I, Lindenbaum M, Rivlin E.: Mean shift tracking with multiple reference color histograms. Computer Vision and Image Understanding. 114, 400-408 (2010) 
Figures

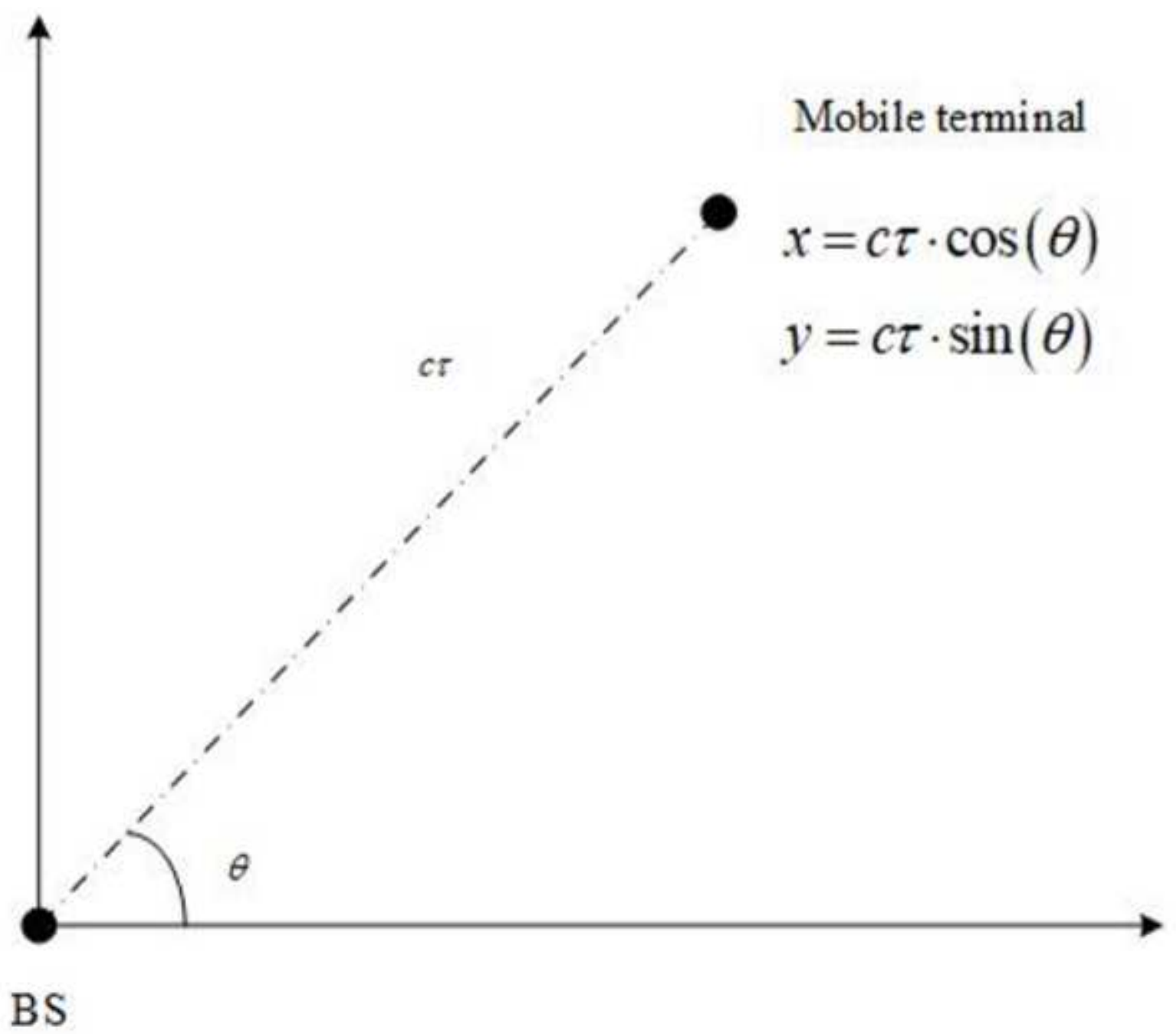

Figure 1

Traditional single station positioning scheme 


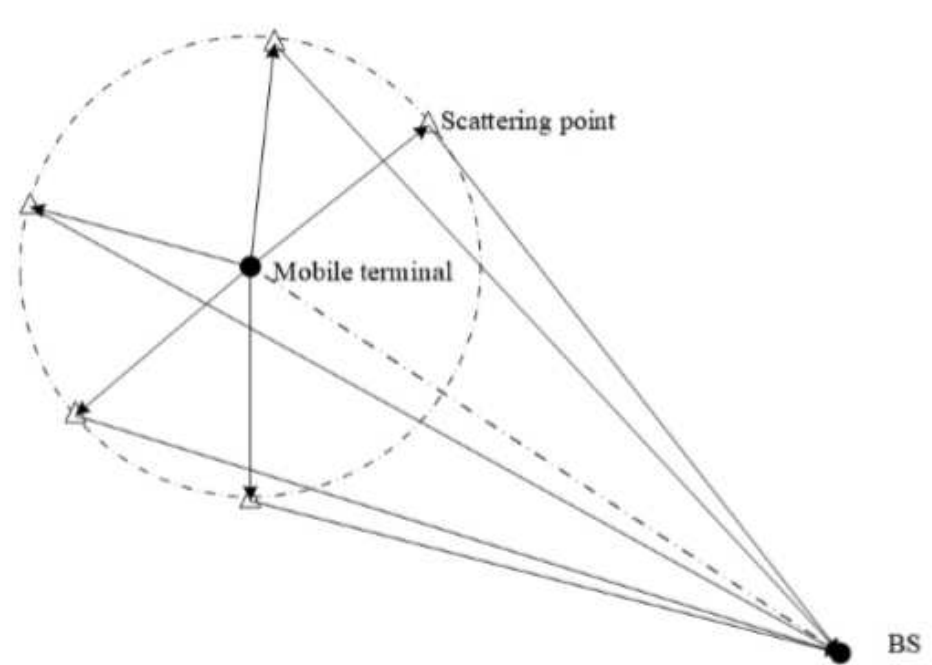

ROS

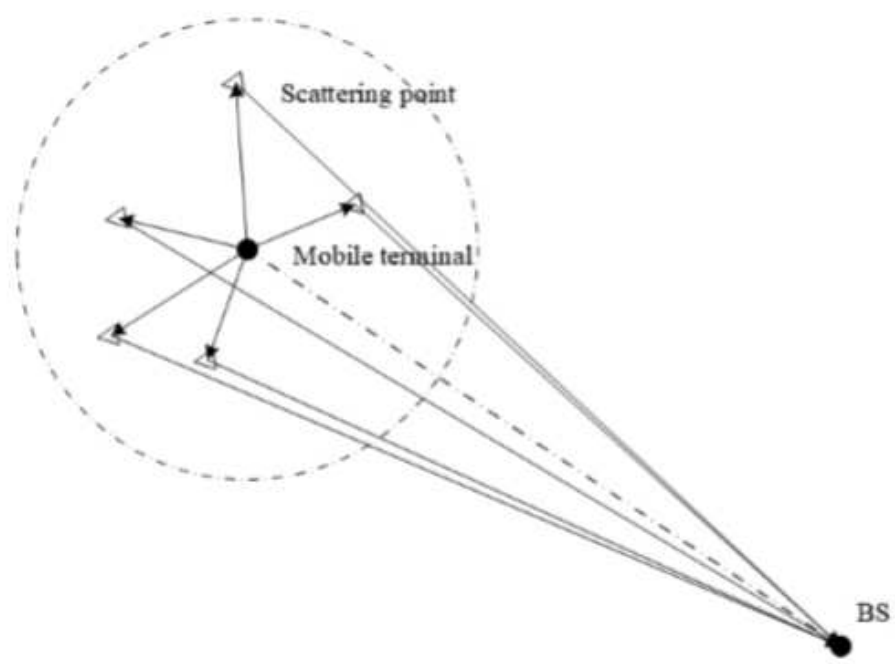

DOS

Figure 2

ROS and DOS

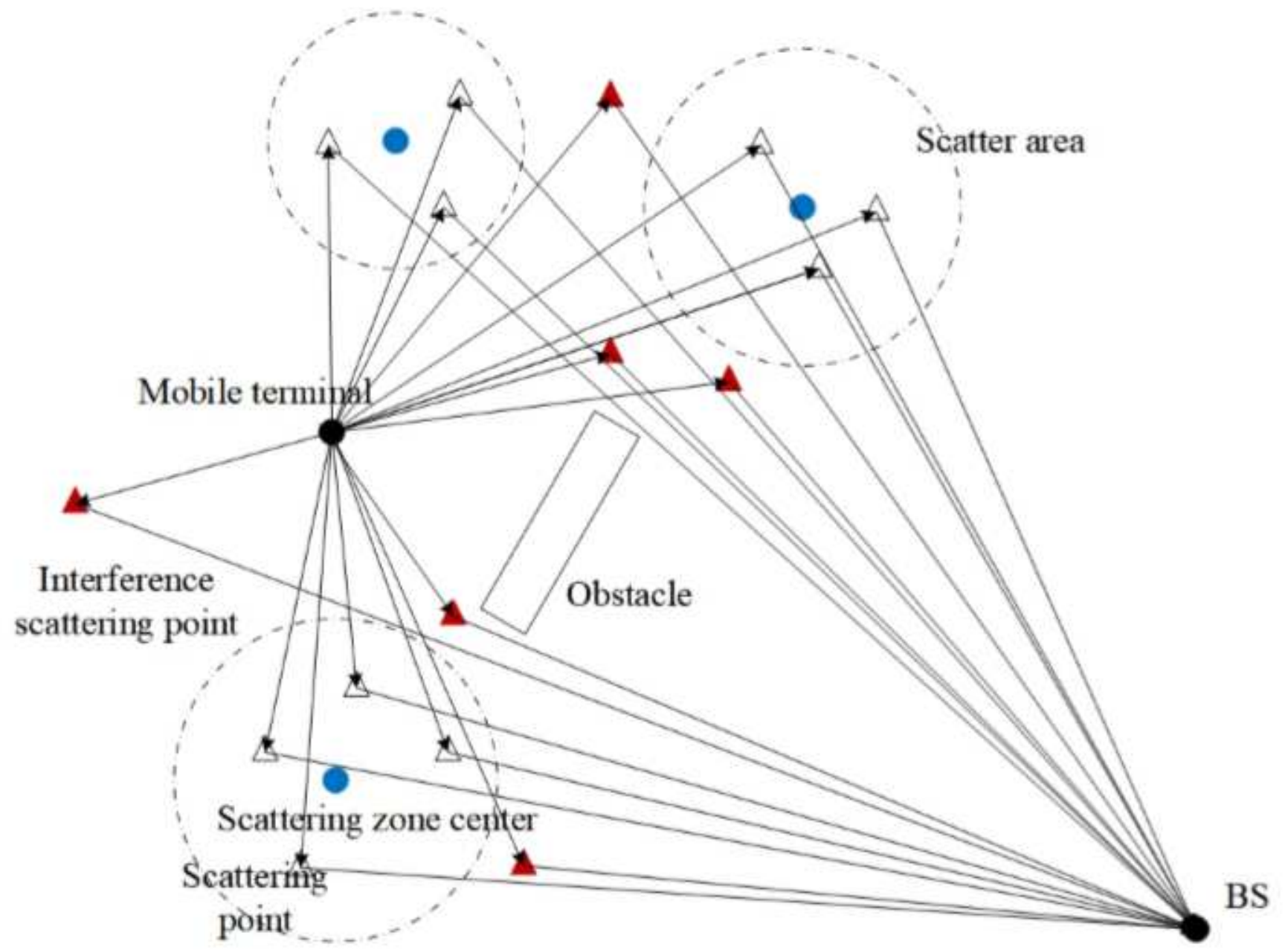

Figure 3 
Scattering area model

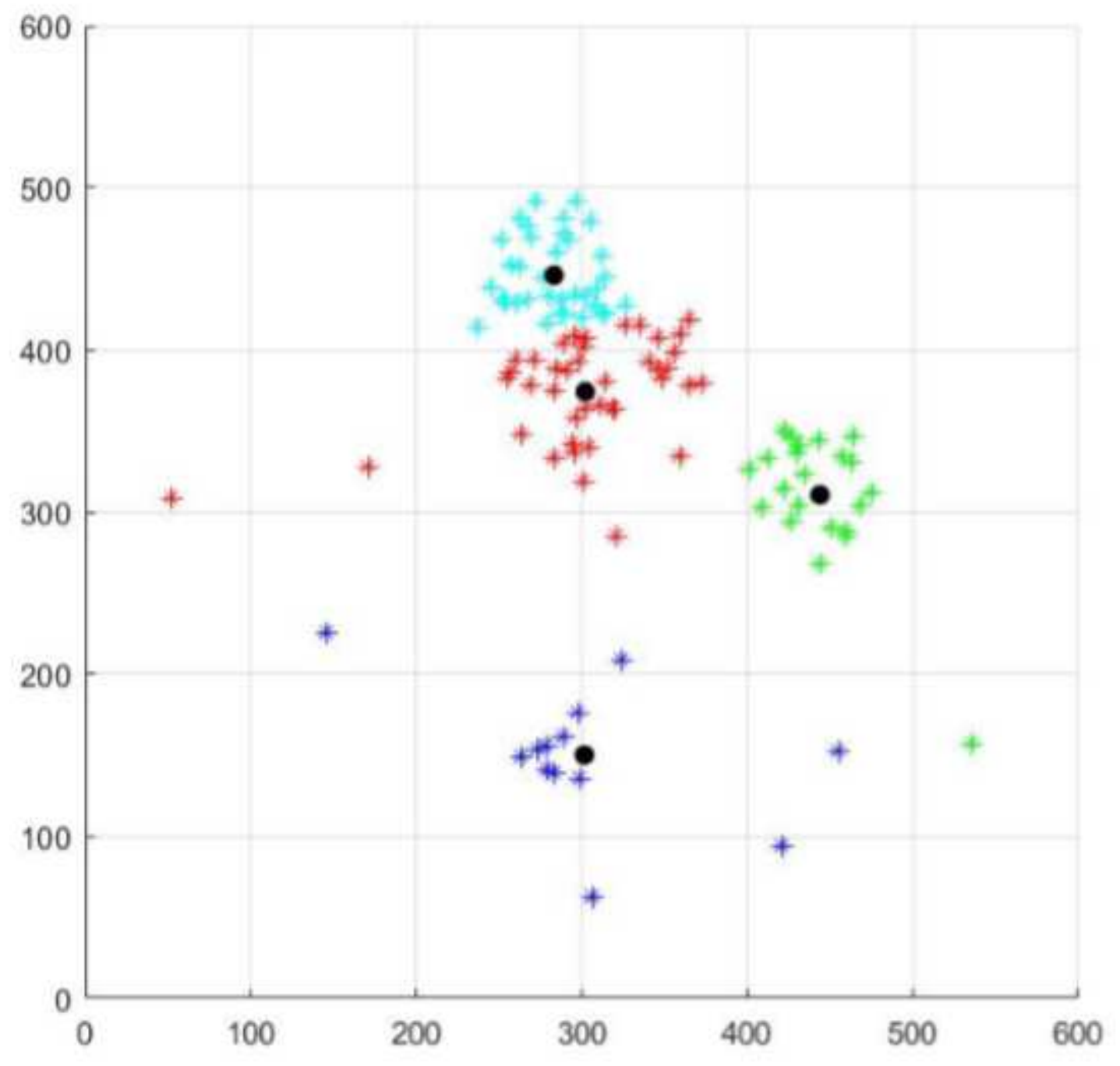

Figure 4

K-means clustering result 


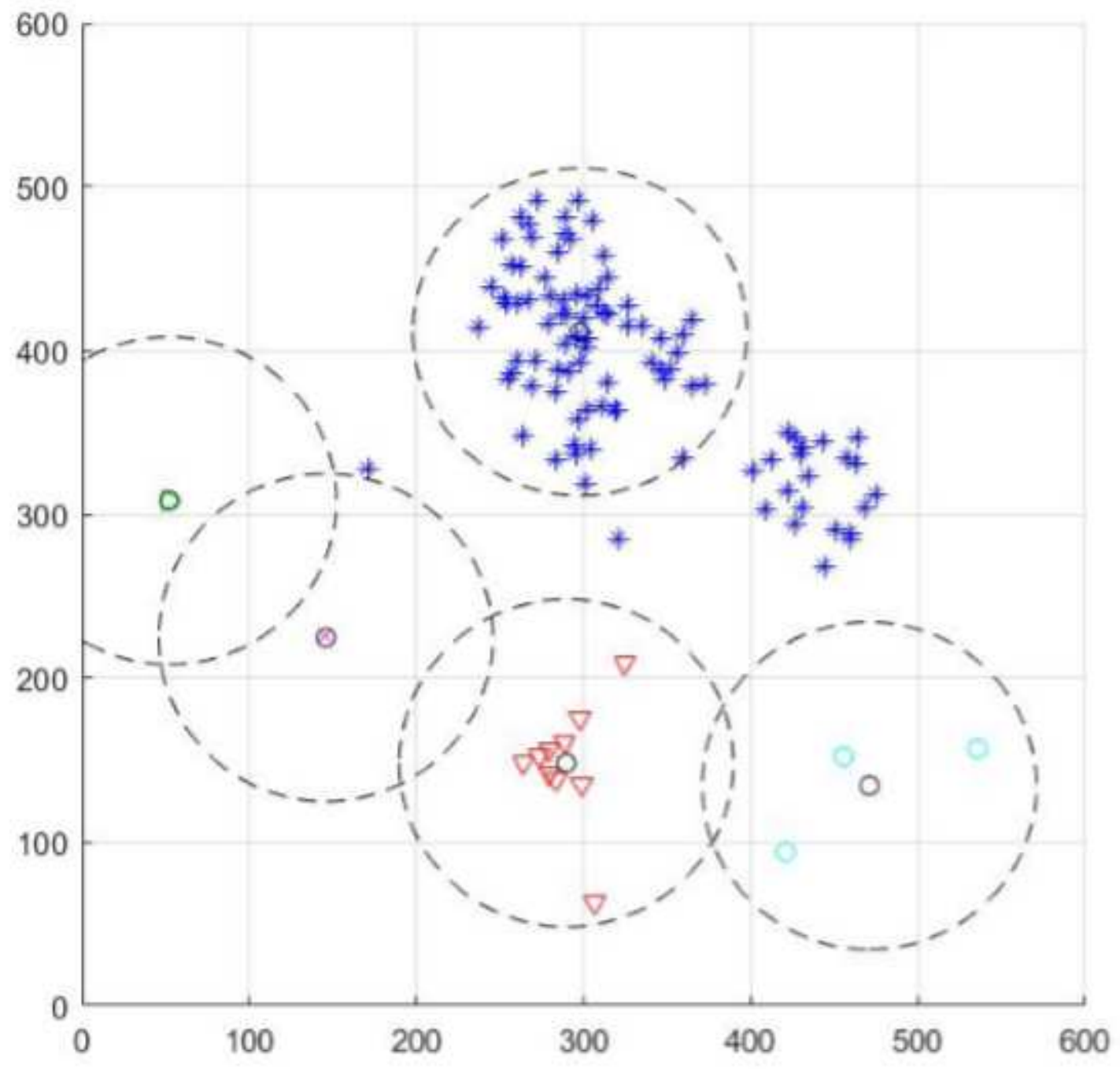

Figure 5

Mean shift clustering result 


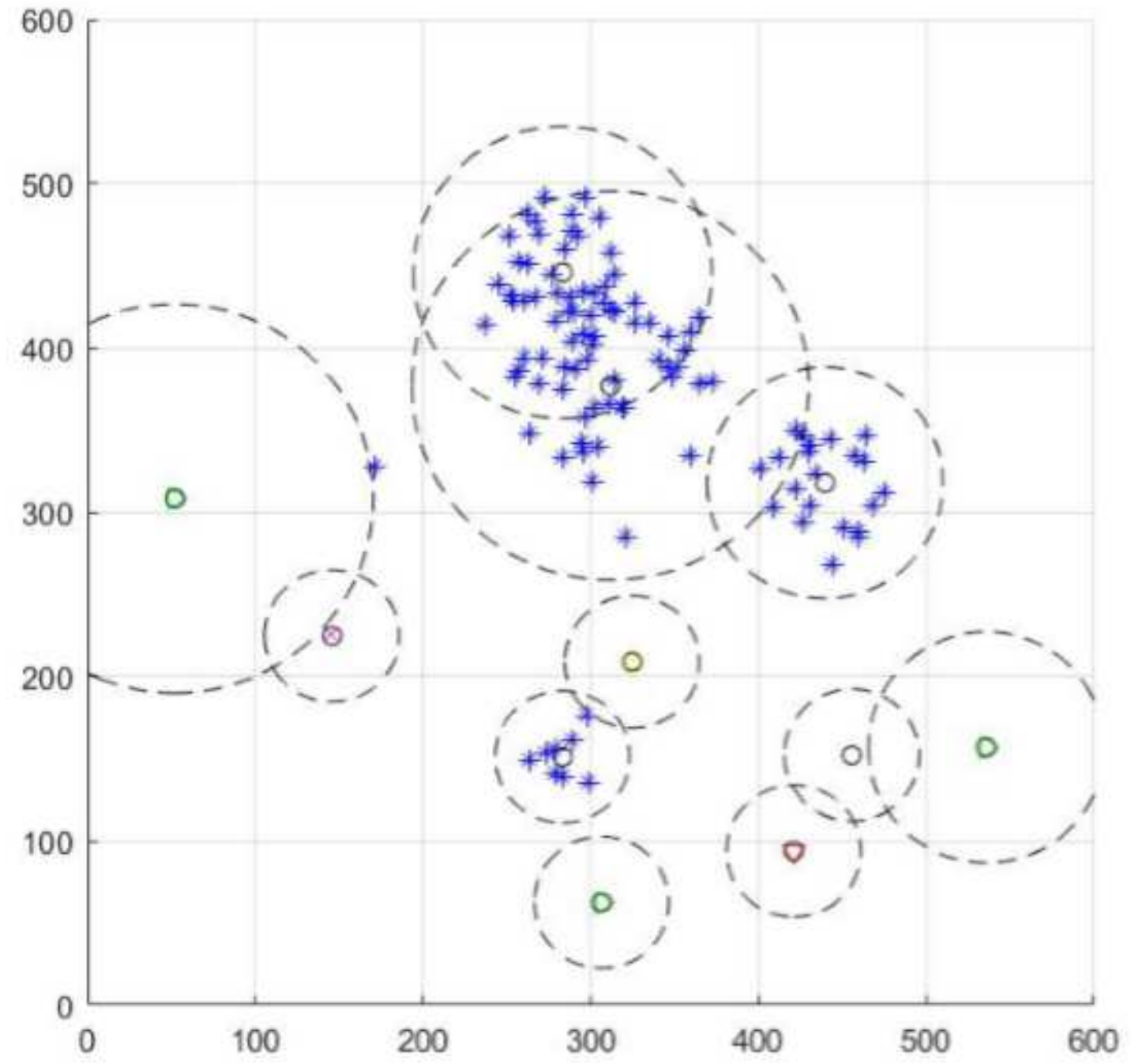

Figure 6

Joint clustering result 


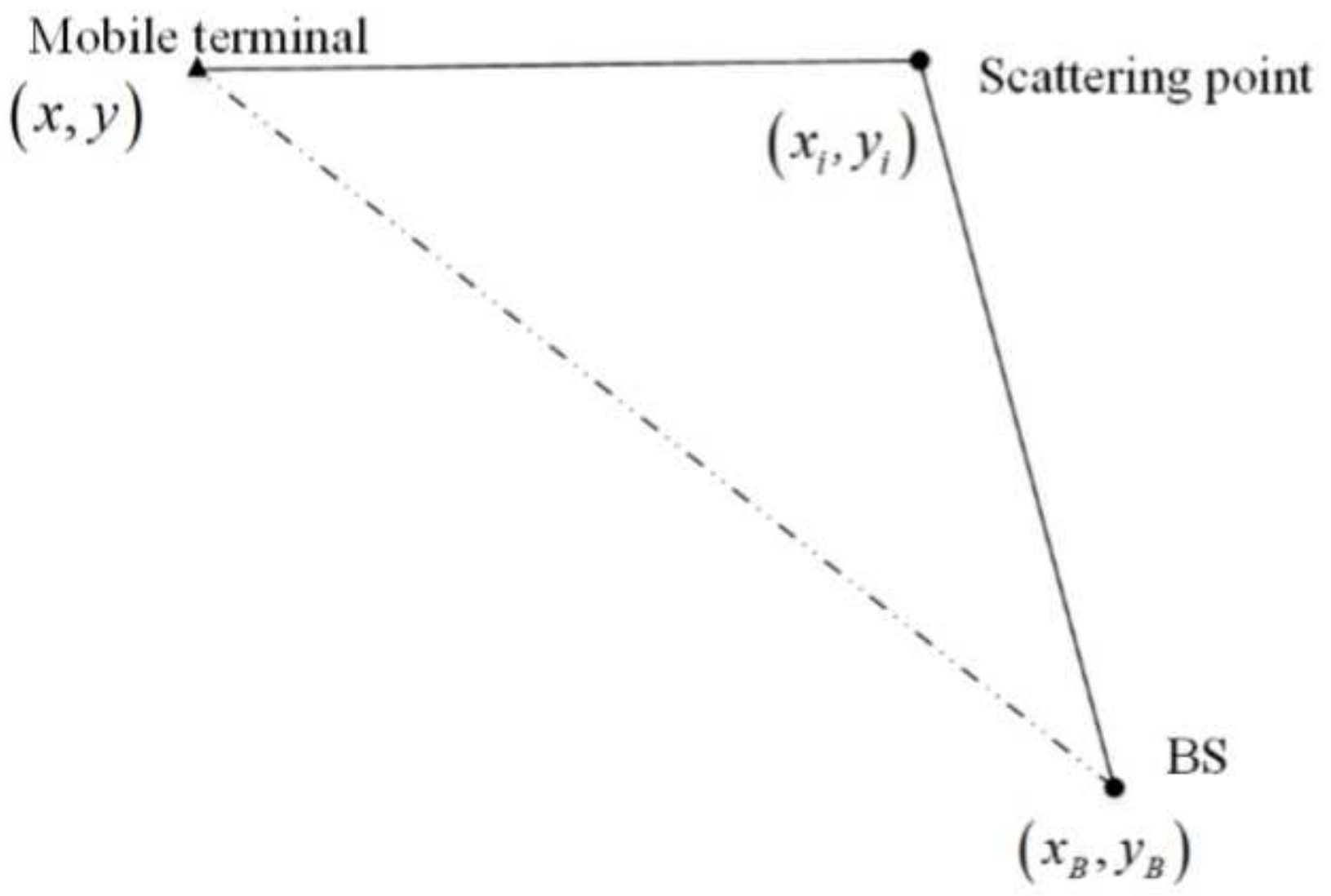

Figure 7

Single station positioning structure in the NLOS 


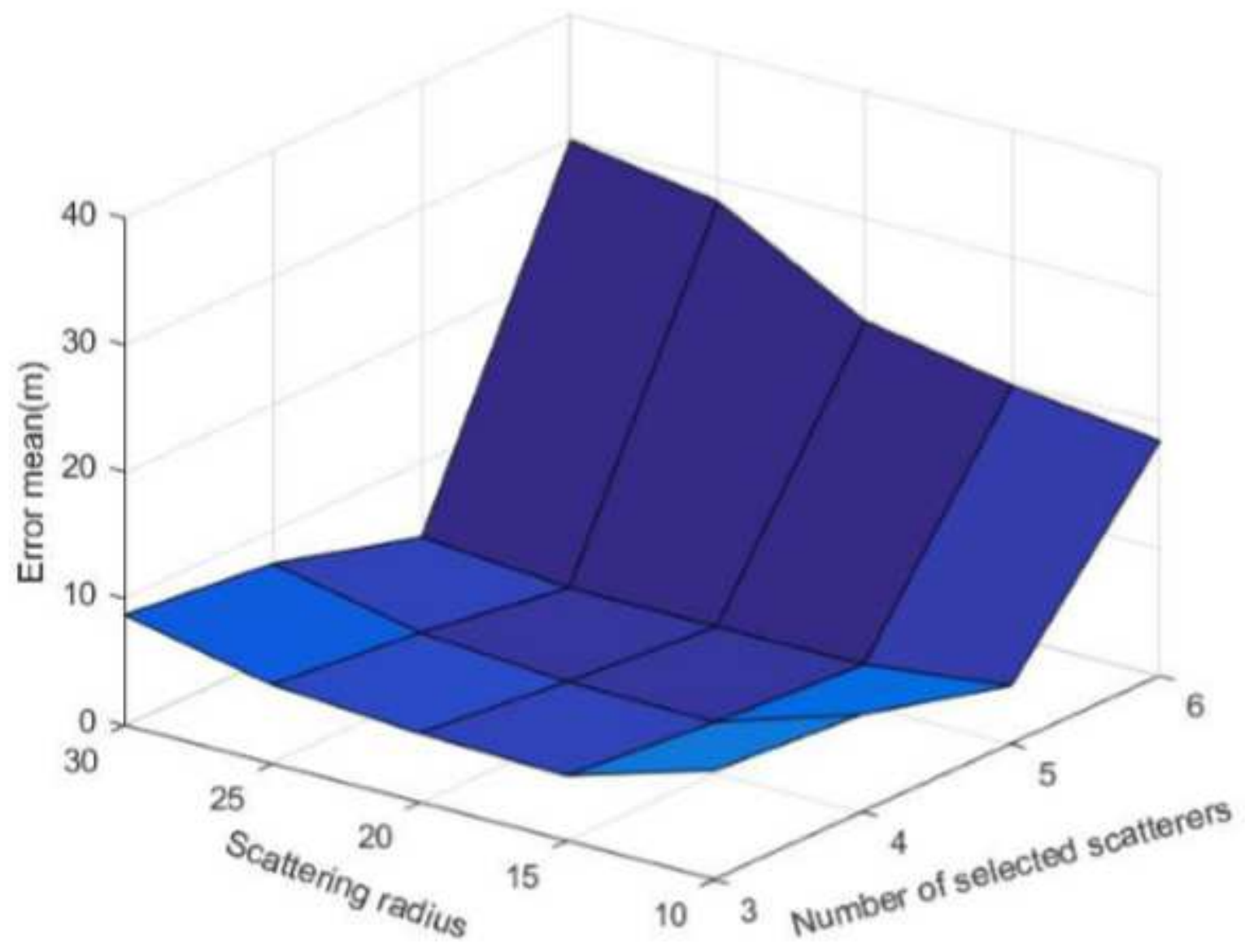

Figure 8

Influence of the number and size of scattering areas on positioning performance 


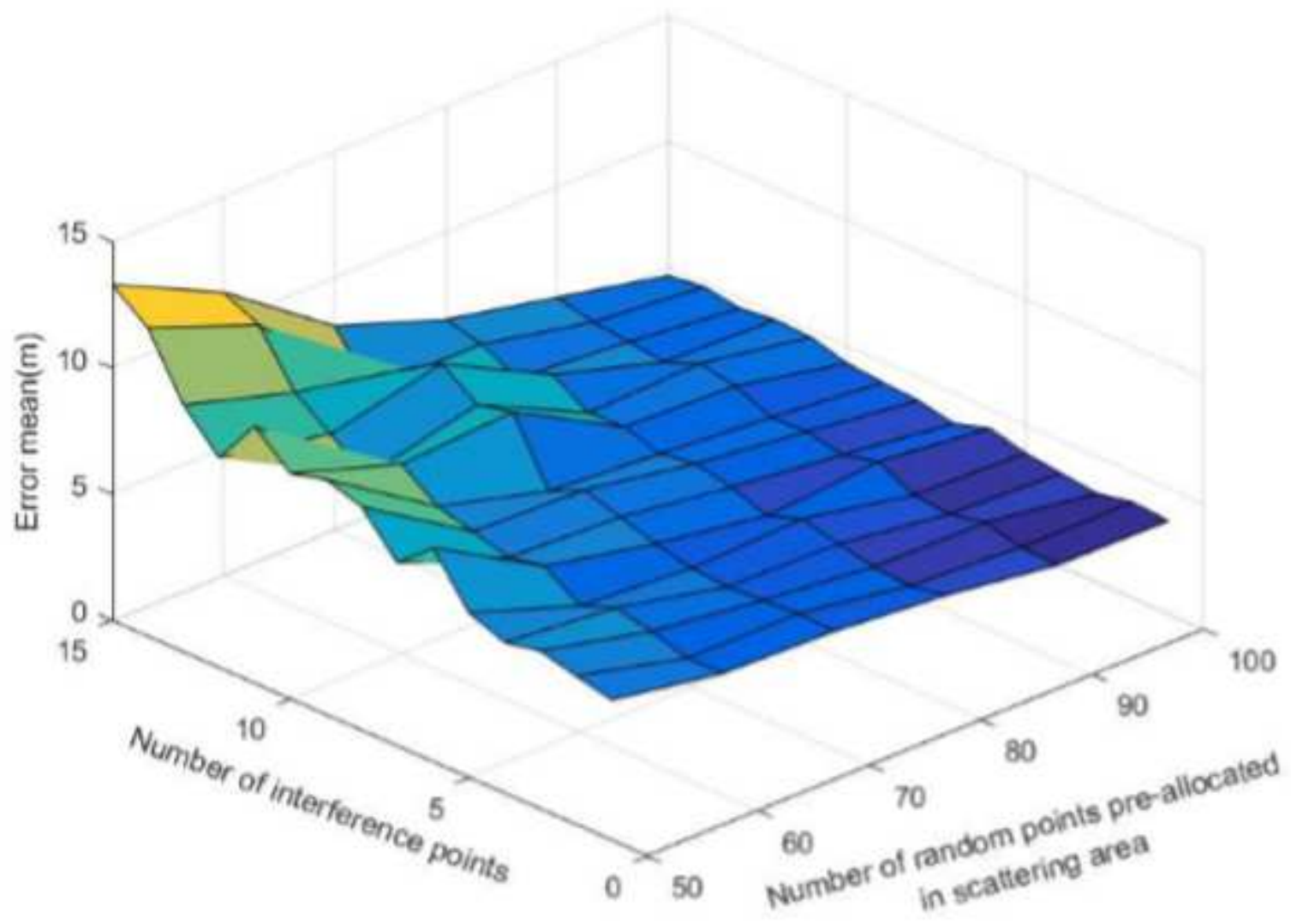

Figure 9

Influence of interference points and pre-assigned random points on the result 


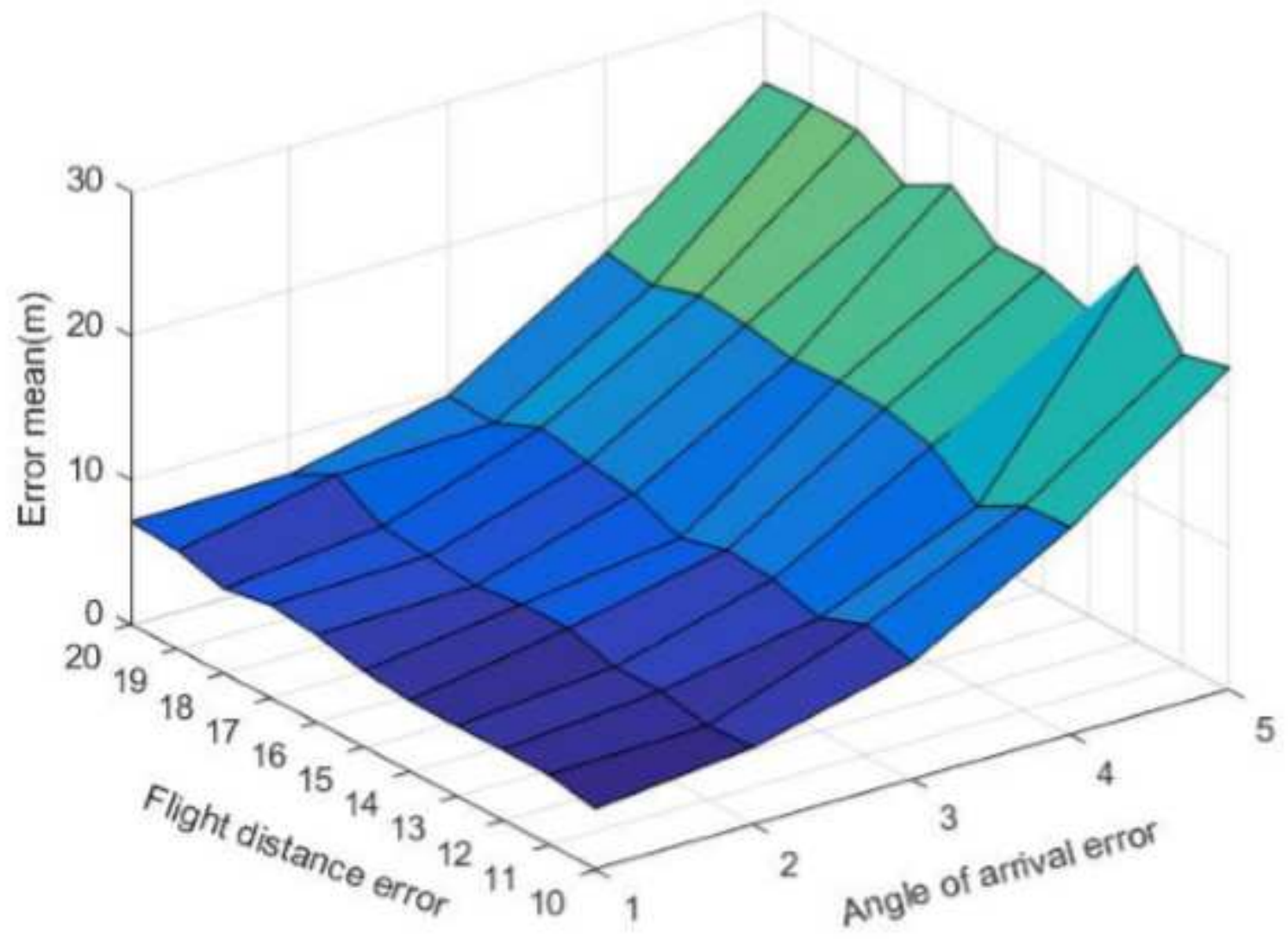

Figure 10

Influence of parameter measurement error on the result 


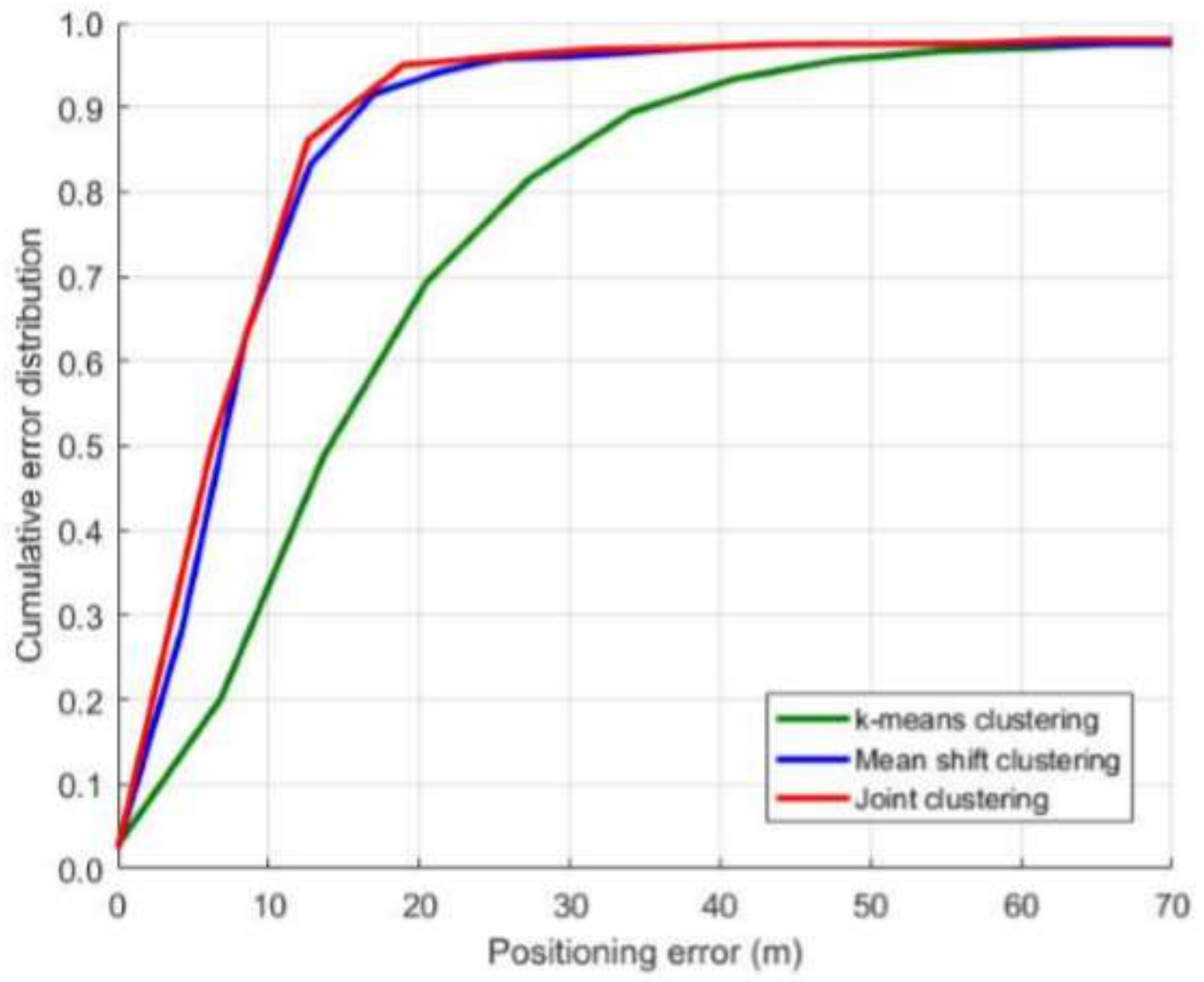

Figure 11

Result by different clustering algorithms 


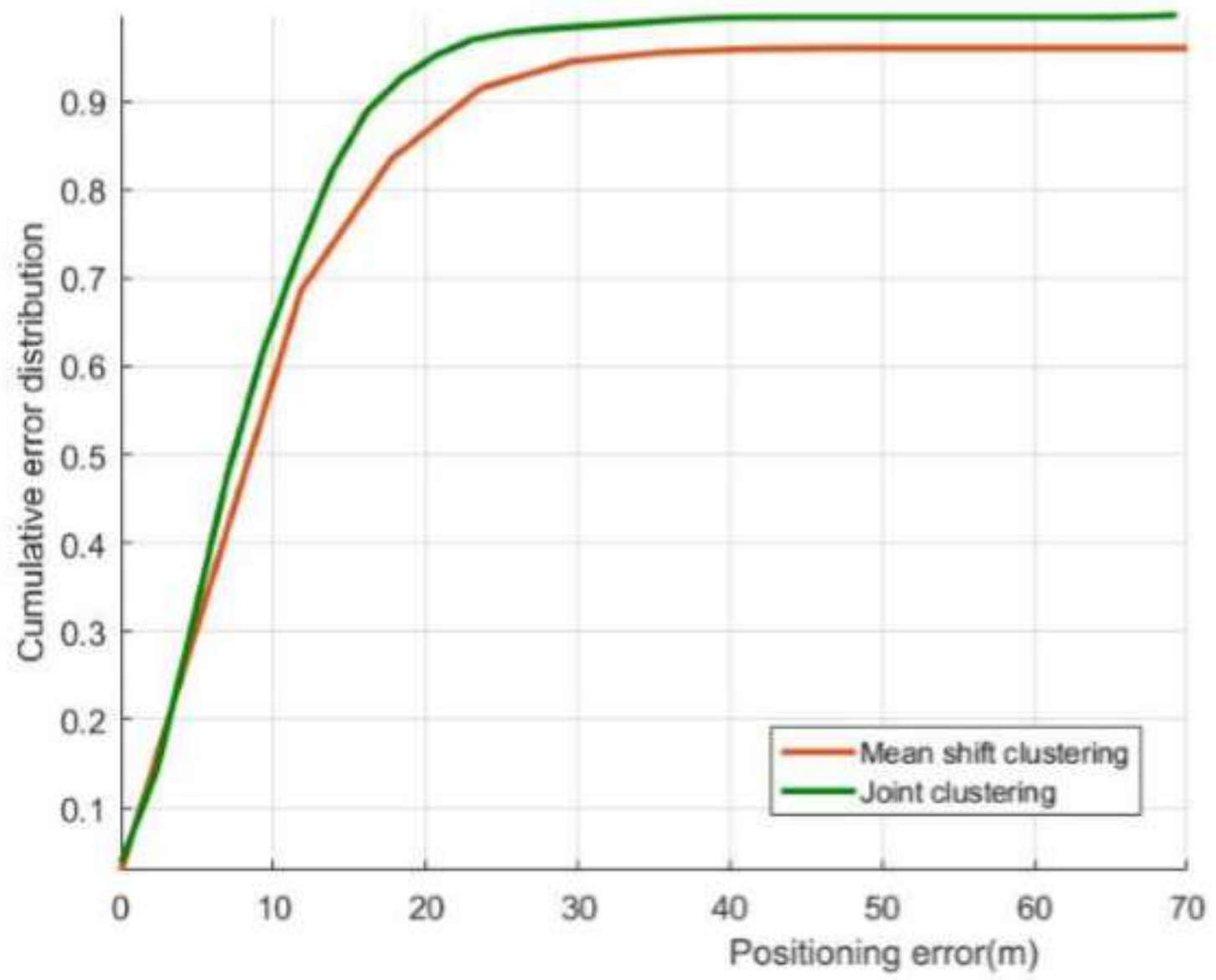

Figure 12

Error CDF based on joint clustering and mean shift clustering when the scattering radius is set randomly. 


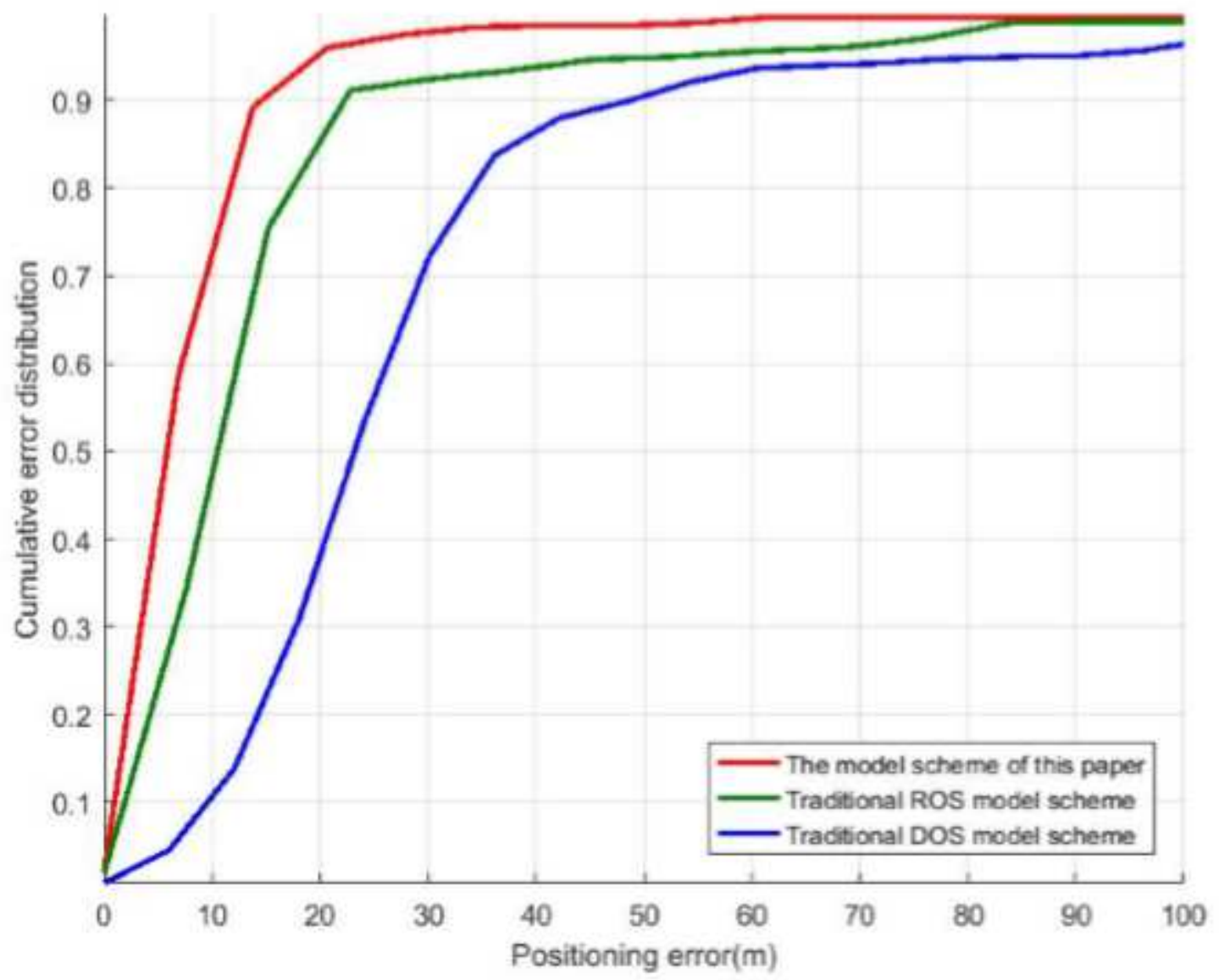

Figure 13

Error CDF of different schemes 


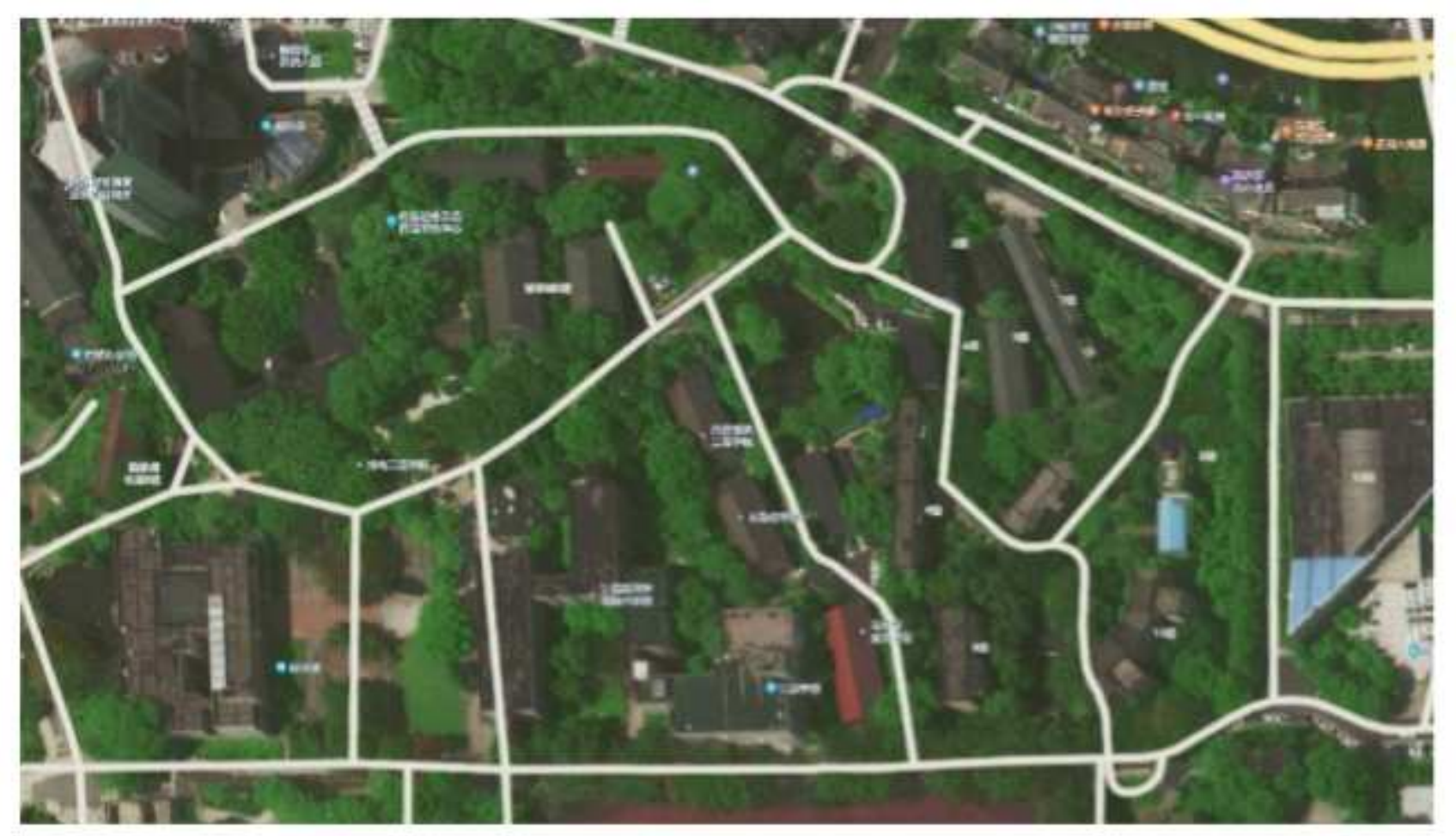

Figure 14

Satellite scan image of simulation scene

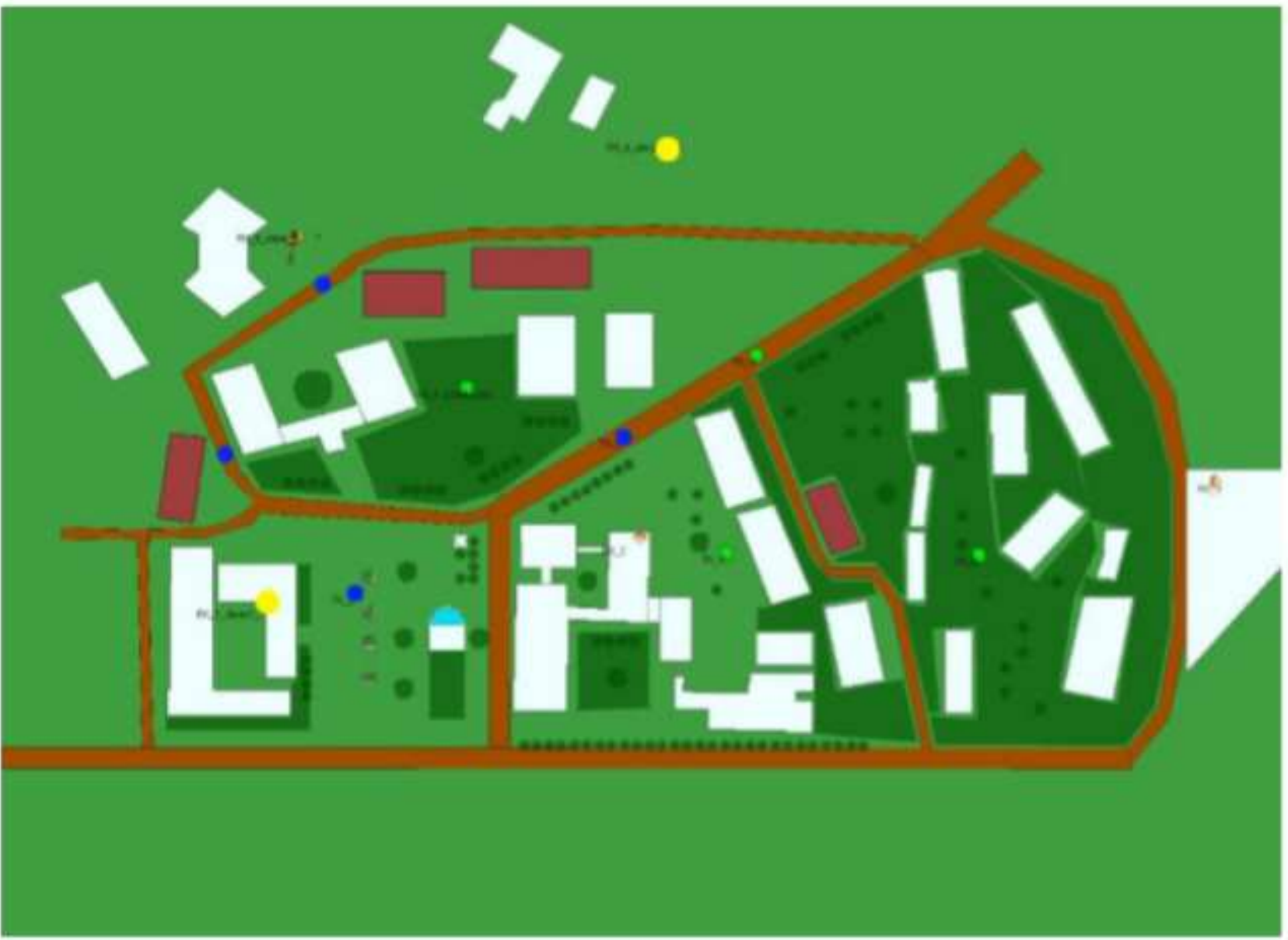


Figure 15

Map model built by the Wireless Insite

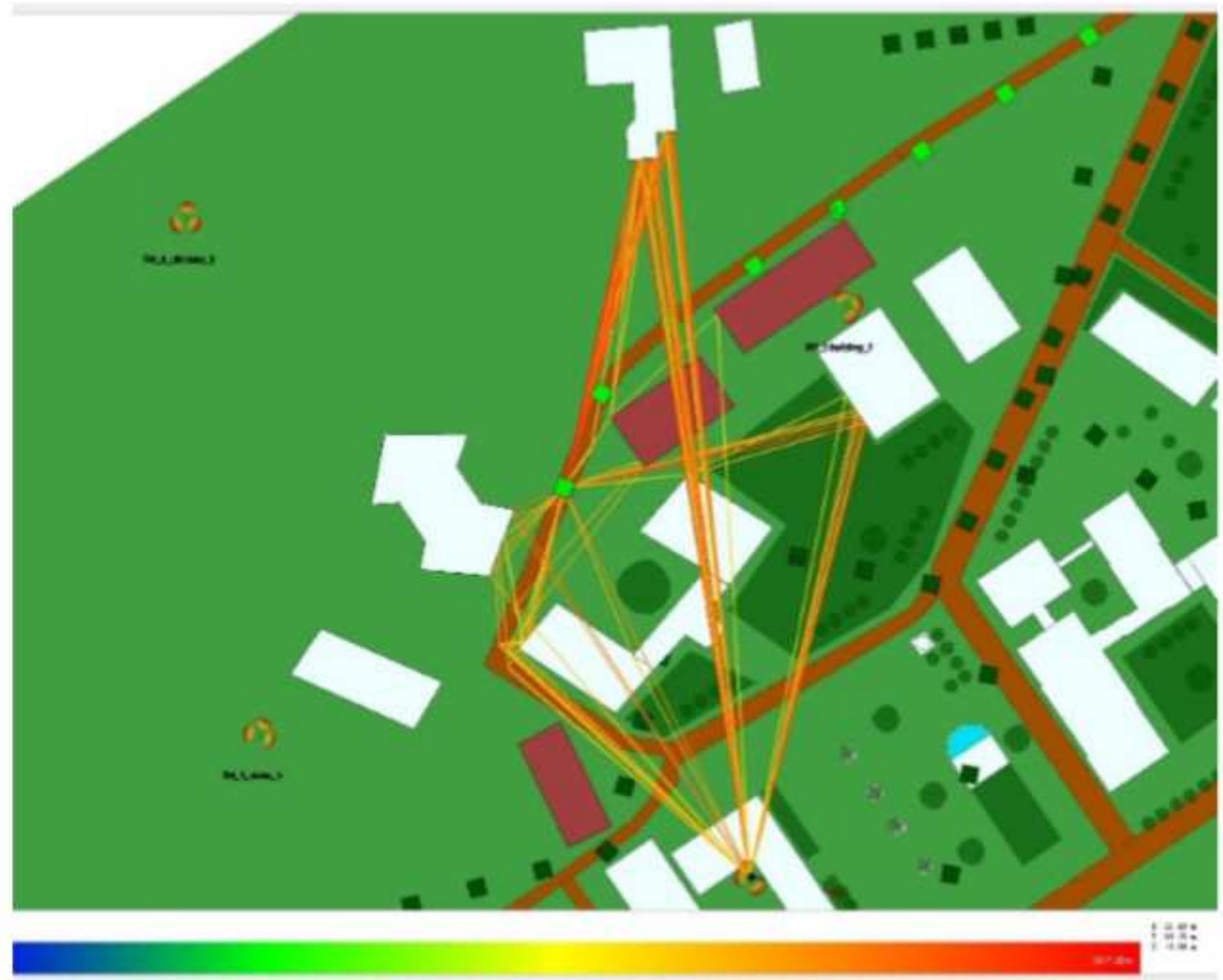

Figure 16

Simulation ray by the Wireless Insite 


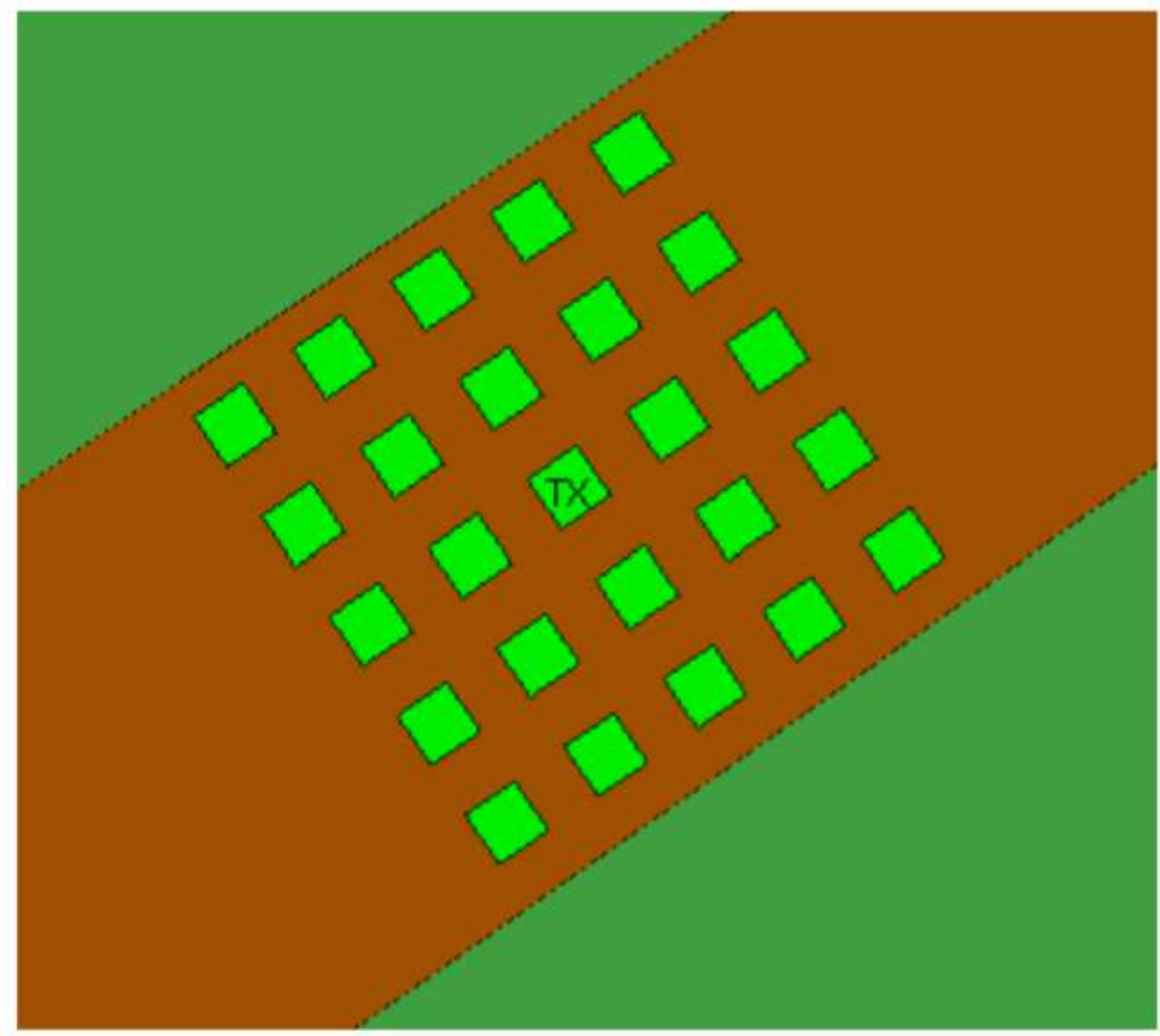

Figure 17

Schematic diagram of sampling points 


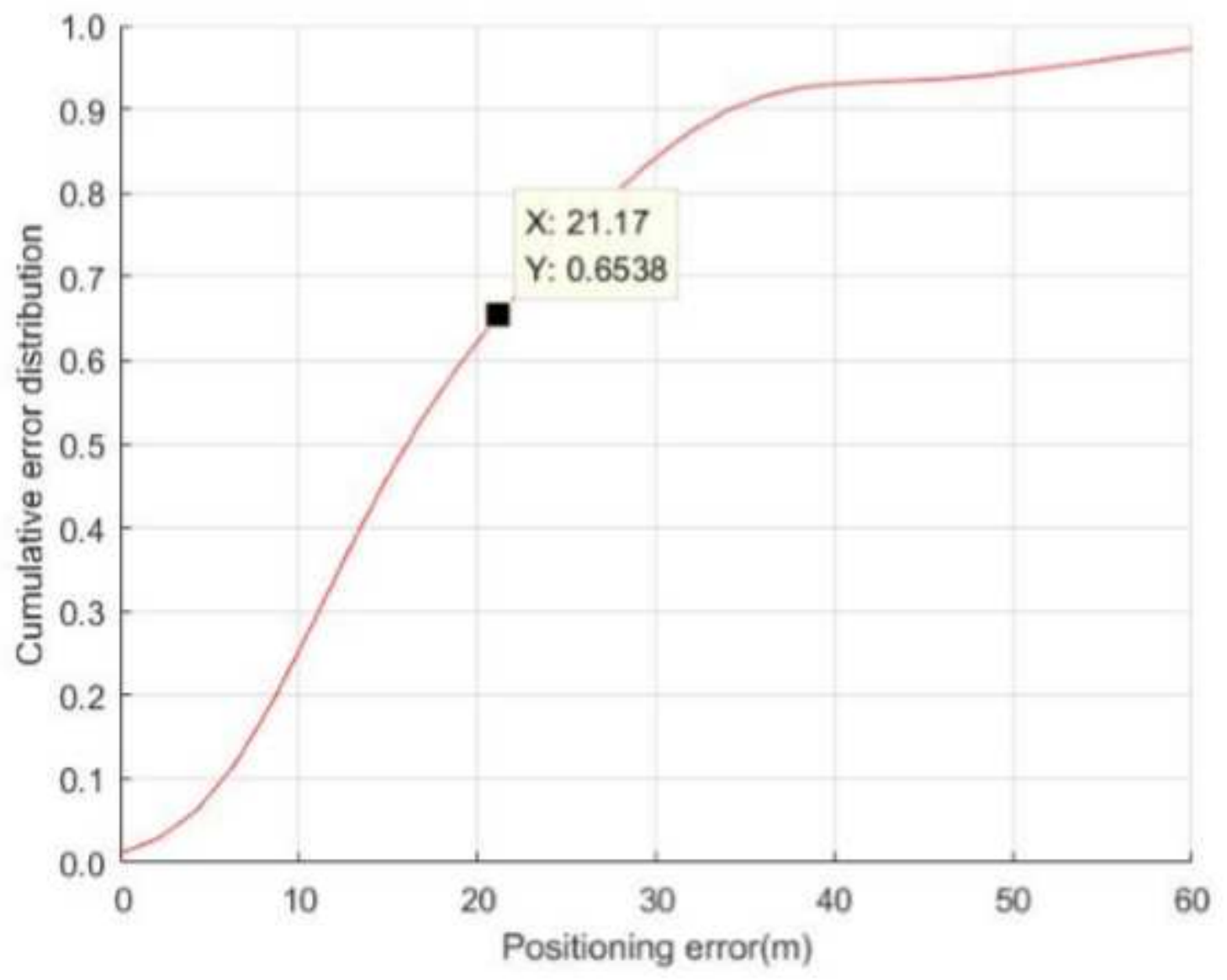

Figure 18

Wireless Insite simulation results 